\title{
Non-associative substructural logics and their semilinear extensions: axiomatization and completeness properties
}

\author{
Petr Cintula, Rostislav Horčík \\ Institute of Computer Science, Academy of Sciences of the Czech Republic \\ Pod vodárenskou věží 2, 18207 Prague, Czech Republic
}

Carles Noguera

Artificial Intelligence Research Institute (IIIA - CSIC)

Campus de la Universitat Autònoma de Barcelona s/n, 08193 Bellaterra, Catalonia, Spain

May 24, 2012

\begin{abstract}
Substructural logics extending the full Lambek calculus FL have largely benefited from a systematical algebraic approach based on the study of their algebraic counterparts: residuated lattices. Recently, a non-associative generalization of FL (which we call SL) has been studied by Galatos and Ono as the logics of lattice-ordered residuated unital groupoids.

This paper is based on an alternative Hilbert-style presentation for SL which is almost (MP)-based. This presentation is then used to obtain, in a uniform way applicable to most (both associative and non-associative) substructural logics, a form of local deduction theorem, description of filter generation, and proper forms of generalized disjunctions.

A special stress is put on semilinear substructural logics (i.e. logics complete w.r.t. linearly ordered algebras). Axiomatizations of the weakest semilinear logic over SL and other prominent substructural logics are provided and their completeness with respect to chains defined over the real unit interval is proved.
\end{abstract}

Keywords: Substructural logics, non-classical logics, non-associative full Lambek logic, latticeordered residuated unital groupoids, deduction theorems, Mathematical Fuzzy Logic, standard completeness

\section{Introduction}

Substructural logics form a wide family of non-classical logics that can be roughly defined as those logical systems such that, when presented by means of a Gentzen-style calculus, lack some of the structural rules, i.e. rules not involving any connective of the language (see e.g. [29, 31, $32]$ ). As such they encompass a variety of systems independently developed since mid XXth century, including relevant logics [1] or many-valued logics like monoidal logic [22] (not satisfying contraction), linear logic [20] (which, besides contraction, also fails to enjoy weakening) or Lambek calculus [26] (which, besides the former two, does not satisfy exchange either). The study of such heterogenous landscape has greatly benefited of a uniform approach, developed in the last two decades in the tradition of Algebraic Logic, which deals with substructural logics as logics of residuated lattices, i.e. propositional logics algebraizable in the sense of [3] whose equivalent algebraic semantics are classes of lattice-ordered residuated monoids (called residuated lattices for short). The weakest logic considered in this line of research is the full Lambek logic FL, whose equivalent algebraic semantics is the variety of all residuated lattices. Most results on the algebraic study of FL and its extensions are collected in the monograph [18]. 
These systematical efforts, nevertheless, have neglected the study of systems lacking another important structural rule: associativity. Indeed, FL does satisfy associativity and for this reason its algebraic semantics interprets (multiplicative) conjunction by a monoidal operation. Actually, there have been several studies on non-associative substructural logics, starting with the original Lambek non-associative calculus [27] (without lattice connectives), and followed (in the full language) e.g. by Buszkowski and Farulewski in [6]. In the recent paper [19] Galatos and Ono introduce a Gentzen-style and a Hilbert-style calculus for the non-associative version of the Full Lambek calculus. They prove that it is an algebraizable logic with the variety of lattice-ordered residuated unital groupoids as its equivalent algebraic semantics; and thus they obtain a natural generalization of the approach used for FL and its extensions. In this paper we work with the bounded extension of this logic, denoted as SL.

Building on this, in [12] a general algebraic framework to deal with substructural logics with SL as the base logic is presented. The authors introduce, as a crucial tool, the notion of almost (MP)based logic: a logic with a Hilbert-style presentation where modus ponens is the only binary rule, there are no rules with more than two premises, and all unary rules are of the form $\varphi \vdash \gamma(\varphi)$, for $\gamma \in \mathrm{DT}$, where the set of terms DT satisfies a natural technical condition. They prove that every almost (MP)-based substructural logic enjoys a local deduction theorem and a certain form of Proof by Cases Property (PCP), which can arguably be seen as the defining property of a reasonable generalized notion of disjunction (as studied by Abstract Algebraic Logic). From this, one can extract a number of interesting consequences for logical systems in general (see [10, 13]) and for substructural logics in particular (see [12]), such as (parameterized) local deduction theorem, description of intersection of filters, axiomatization of logics given by positive universal classes of algebras, or axiomatization of intersection of axiomatic extensions of a given logic. It was shown in [12] that FL, and hence all its axiomatic extensions, are indeed almost (MP)-based and, therefore, the authors could apply their general theory to all these logics and obtain, in a uniform way, the mentioned consequences. However, the problem was left open for SL and other non-associative logics.

The main purpose of the present paper is to solve this problem. Indeed we present an alternative Hilbert-style axiomatization of SL which, besides modus ponens, has the following unary rules: ${ }^{1}$

$$
\begin{array}{cl}
\left(\operatorname{Adj}_{\mathrm{u}}\right) & \varphi \vdash \varphi \wedge \overline{1} \\
(\alpha) & \varphi \vdash \delta \& \varepsilon \rightarrow \delta \&(\varepsilon \& \varphi) \\
\left(\alpha^{\prime}\right) & \varphi \vdash \delta \& \varepsilon \rightarrow(\delta \& \varphi) \& \varepsilon \\
(\beta) & \varphi \vdash \delta \rightarrow(\varepsilon \rightarrow(\varepsilon \& \delta) \& \varphi) \\
\left(\beta^{\prime}\right) & \varphi \vdash \delta \rightarrow(\varepsilon \rightsquigarrow(\delta \& \varepsilon) \& \varphi)
\end{array}
$$

We show that this axiomatization is indeed almost (MP)-based which allows us (as mentioned above) to naturally extend to the non-associative case many results so far only known for associative logics. This clearly demonstrates that the algebraic approach started in [19] and [12] is the right generalization of that used for associative logics in [18].

Among others we obtain a method to find an axiomatization of the minimum logic $\mathrm{L}^{\ell}$ extending a given logic $\mathrm{L}$ which is complete with respect to linearly ordered L-algebras (sometimes simply called L-chains). The class of logics complete with respect to chains has been introduced in a very general framework in [11] under the name semilinear logics. ${ }^{2}$ When restricted to the framework of substructural logics, semilinear logics form a distinctive subfamily that contains most systems referred to in the literature as fuzzy logics. The discipline that studies these systems, Mathematical Fuzzy Logic [9], has shown an interest in considering increasingly weaker systems, aiming to find the basic fuzzy logic contained in all others.

\footnotetext{
${ }^{1}$ The rules $(\alpha)$ and $(\beta)$ are taken from corresponding algebraic terms that appeared in [4] where, in the context of the study of a non-associative version of Hájek's logic BL, they were used to describe filters in commutative integral lattice-ordered residuated unital groupoids. To cope with the lack of exchange and weakening (i.e. lack of commutativity and integrality in the algebras), we also need to consider unit-adjunction and a modified version of those rules: $\left(\alpha^{\prime}\right)$ and $\left(\beta^{\prime}\right)$.

${ }^{2}$ The term semilinear refers to the fact that these logics can be equivalently characterized as those logics such that their (relatively) subdirectly irreducible algebras are linearly ordered.
} 
Several systems have been proposed as such and later replaced by weaker ones, for instance: Hájek's logic BL $[21,7], \mathrm{FL}_{\mathrm{ew}}^{\ell}=\mathrm{MTL}[14,24], \mathrm{FL}_{\mathrm{w}}^{\ell}=\mathrm{psMTL}^{r}[25]$, and $\mathrm{FL}_{\mathrm{e}}^{\ell}=\mathrm{UL}[28] .{ }^{3}$ One can observe that the common feature of all the mentioned logics is that they enjoy a standard completeness theorem, i.e. completeness with respect to a semantics of algebras defined on the real unit interval $[0,1]$, which is implicitly regarded by many authors (and sometimes even explicitly e.g. in [28]) as an essential requirement for fuzzy logics. Interestingly enough, the logic $\mathrm{FL}^{\ell}$ of FL-chains does not enjoy standard completeness (see [33]), therefore, for these authors it can hardly be taken as a good candidate for a really basic fuzzy logic (even though for some it is fuzzy enough [2]). Moreover, one can also argue that $\mathrm{FL}^{\ell}$ is still not basic enough because it satisfies a remaining structural rule: associativity. This brings us again to the main motivation of this paper, the algebraic study of non-associative logics, and now also the study of their semilinear extensions. Following the methods and results in $[12,23]$ for semilinear associative substructural logics, the second aim of the present paper, thus, is to use the terms appearing in almost (MP)based presentations to obtain axiomatizations of semilinear non-associative substructural logics and study their standard completeness properties. In particular, we obtain a presentation of $\mathrm{SL}^{\ell}$ and prove that it enjoys completeness with respect to residuated unital groupoids over $[0,1]$. Therefore $\mathrm{SL}^{\ell}$ can be seen as a new good candidate for a basic fuzzy logic, for it is really basic (it does not even satisfy associativity) and really fuzzy (it is standard complete).

Structure of the paper In Section 2 we briefly recall the necessary syntactical and semantical preliminaries for the paper: 2.1 shows the Hilbert-style presentation of SL given by Galatos and Ono, lists some important syntactical properties that hold in the system and introduces prominent axiomatic extensions, while 2.2 introduces the semantics for these logics based on lattice-ordered residuated unital groupoids. Section 3 is devoted to the general algebraic study of (non-associative) substructural logics. Subsection 3.1 presents an alternative Hilbert-style axiomatic system for SL (the formal proof of the equivalence of this new system with the original one can be found in Appendix A) and uses it to show that it is an almost (MP)-based logic. Subsection 3.2 derives from this result a form of (parameterized) local deduction theorem for SL and its extensions and some results on filter generation. Subsection 3.3 extracts from the terms appearing in almost (MP)based presentation a description of a p-disjunction for SL, shows its simplifications in prominent extensions, and considers the aforementioned applications of these p-disjunctions. Section 4 is devoted to semilinear extensions of non-associative substructural logics, i.e. the logics given by their linearly ordered algebras. Subsection 4.1, as yet another application of almost (MP)-basedness and p-disjunctions, shows several equivalent ways to axiomatize these semilinear logics. Finally, Subsection 4.2 gives, by means of algebraic constructions, a proof of completeness of SL and other non-associative logics with respect to their chains defined over the real and the rational unit intervals.

\section{Preliminaries}

\subsection{Syntactical properties}

The weakest logic we consider in this paper is the bounded version of the non-associative full Lambek calculus studied by Galatos and Ono in [19]. We will call it SL and formulate it in the language $\mathcal{L}_{\mathrm{SL}}=\{\wedge, \vee, \&, \rightarrow, \rightsquigarrow, \overline{0}, \overline{1}, \perp, \top\}$ (we also make use of the defined connective $\varphi \leftrightarrow \psi=$ $(\varphi \rightarrow \psi) \wedge(\psi \rightarrow \varphi))$. When writing formulae in this language we will assume that the increasing binding order of connectives is: first \&, then $\{\wedge, \vee\}$, and finally $\{\rightarrow, \rightsquigarrow\}$. This logic can be axiomatized by means of the following Hilbert-style calculus presented in Table 1 (it is obtained from that of [19, Figure 5] by expanding its language with a new basic connective $\perp$ and derived connective $T$ defined as $\perp \rightarrow \perp$ and by adding the axiom $\perp \rightarrow \varphi$ ).

\footnotetext{
${ }^{3}$ An alternative path in the search for weaker systems, instead of removing logical laws, has consisted in restricting the language by considering fragments of fuzzy logics (see e.g. $[15,8]$ ).
} 


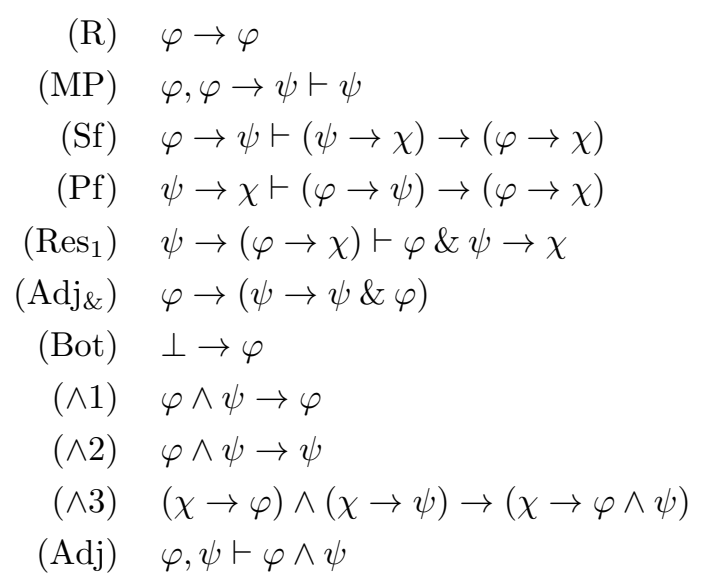

Table 1: Axiomatic system of SL

Galatos and Ono also provide in [19] a Gentzen-style calculus which can be easily extended to a calculus for SL. On the other hand, SL is implicitly presented by an alternative Hilbertstyle system in [12, Definition 2.5.1]. By using any of these presentations, one may obtain other well-known properties of substructural logics which already hold in SL:

$$
\begin{aligned}
(\mathrm{T}) & \chi \rightarrow \varphi, \varphi \rightarrow \psi \vdash \chi \rightarrow \psi \\
(\mathrm{Pf})_{\rightsquigarrow} & \psi \rightarrow \chi \vdash(\varphi \rightsquigarrow \psi) \rightarrow(\varphi \rightsquigarrow \chi) \\
\left(\mathrm{Adj}_{\mathrm{u}}\right) & \varphi \vdash \varphi \wedge \overline{1} \\
\left(\mathrm{E}_{\rightsquigarrow 2}\right) & \psi \rightarrow(\varphi \rightsquigarrow \chi) \vdash \varphi \rightarrow(\psi \rightarrow \chi)
\end{aligned}
$$

$\left(\mathrm{Symm}_{2}\right) \quad \varphi \rightarrow \psi \vdash \varphi \rightsquigarrow \psi$

$$
\left(\operatorname{Res}_{2}\right) \quad \varphi \& \psi \rightarrow \chi \vdash \psi \rightarrow(\varphi \rightarrow \chi)
$$

We also list some other properties that will be useful later; $\left(\mathrm{P}_{\mathrm{SL}} 2\right)-\left(\mathrm{P}_{\mathrm{SL}} 24\right)$ are taken from [12, Proposition 2.5.5] (where one can find their proofs), the remaining ones can be proved easily (e.g. in the Gentzen calculus for SL).

$$
\begin{array}{ll}
\left(\mathrm{P}_{\mathrm{SL}} 2\right) & \vdash \varphi \&(\varphi \rightarrow \psi) \rightarrow \psi \\
\left(\mathrm{P}_{\mathrm{SL}} 8\right) & \varphi \rightarrow \psi \vdash \chi \& \varphi \rightarrow \chi \& \psi \\
\left(\mathrm{P}_{\mathrm{SL}} 9\right) & \varphi \rightarrow \psi \vdash \varphi \& \chi \rightarrow \psi \& \chi \\
\left(\mathrm{P}_{\mathrm{SL}} 10\right) & \varphi_{1} \rightarrow \psi_{1}, \varphi_{2} \rightarrow \psi_{2} \vdash \varphi_{1} \& \varphi_{2} \rightarrow \psi_{1} \& \psi_{2} \\
\left(\mathrm{P}_{\mathrm{SL}} 20\right) & \vdash \chi \&(\varphi \vee \psi) \leftrightarrow(\chi \& \varphi) \vee(\chi \& \psi) \\
\left(\mathrm{P}_{\mathrm{SL}} 21\right) & \vdash(\varphi \vee \psi) \& \chi \leftrightarrow(\varphi \& \chi) \vee(\psi \& \chi) \\
\left(\mathrm{P}_{\mathrm{SL}} 22\right) & \vdash(\varphi \wedge \overline{1}) \&(\psi \wedge \overline{1}) \rightarrow \varphi \wedge \overline{1} \\
\left(\mathrm{P}_{\mathrm{SL}} 23\right) & \vdash(\varphi \wedge \overline{1}) \&(\psi \wedge \overline{1}) \rightarrow \psi \wedge \overline{1} \\
\left(\mathrm{P}_{\mathrm{SL}} 24\right) & \vdash(\varphi \rightarrow \psi) \wedge \overline{1} \rightarrow(\varphi \wedge \overline{1} \rightarrow \psi \wedge \overline{1}) \\
\left(\mathrm{P}_{\mathrm{SL}} 25\right) & \vdash(\varphi \rightarrow \psi) \wedge \overline{1} \rightarrow(\varphi \vee \chi \rightarrow \psi \vee \chi) \\
\left(\mathrm{P}_{\mathrm{SL}} 26\right) & \vdash(\varphi \rightarrow \psi) \wedge \overline{1} \rightarrow(\varphi \vee \psi \rightarrow \psi) \\
\left(\mathrm{P}_{\mathrm{SL}} 27\right) & \vdash(\psi \rightarrow \varphi) \wedge \overline{1} \rightarrow(\varphi \vee \psi \rightarrow \varphi) \\
\left(\mathrm{P}_{\mathrm{SL}} 28\right) & \vdash \varphi \wedge \overline{1} \rightarrow(\varphi \wedge \overline{1}) \wedge \overline{1}
\end{array}
$$




$\begin{array}{lll}\mathrm{a}_{1} & \varphi \&(\psi \& \chi) \rightarrow(\varphi \& \psi) \& \chi & \text { re-associate to the left } \\ \mathrm{a}_{2} & (\varphi \& \psi) \& \chi \rightarrow \varphi \&(\psi \& \chi) & \text { re-associate to the right } \\ \mathrm{e} & \varphi \& \psi \rightarrow \psi \& \varphi & \text { exchange } \\ \mathrm{c} & \varphi \rightarrow \varphi \& \varphi & \text { contraction } \\ \mathrm{i} \quad \psi \rightarrow(\varphi \rightarrow \psi) & \text { left weakening } \\ \mathrm{o} \quad \overline{0} \rightarrow \varphi & \text { right weakening }\end{array}$

Table 2: Structural rules

Some important extensions of SL are obtained by adding the axioms $\mathrm{a}_{1}, \mathrm{a}_{2}$, e, c, i, o corresponding to structural rules (see Table 2). Given any $S \subseteq\left\{\mathrm{a}_{1}, \mathrm{a}_{2}, \mathrm{e}, \mathrm{c}, \mathrm{i}, \mathrm{o}\right\}$, by $\mathrm{SL}_{S}$ we denote the axiomatic extension of SL by $S$. If $\left\{\mathrm{a}_{1}, \mathrm{a}_{2}\right\} \subseteq S$, then instead of them we write the symbol 'a'. Analogously if $\{\mathrm{i}, \mathrm{o}\} \subseteq S$, instead of them we write the symbol 'w'. Equivalent ways to formulate these axioms may be found e.g. in [12, Theorem 2.5.7].

$\mathrm{SL}_{\mathrm{a}}$ is, in fact, the bounded version of full Lambek logic FL. Thus although in this paper we mainly focus on the study of non-associative substructural logics, we can see that our framework encompasses the associative systems as well. For the sake of simplicity we keep the language fixed and we only consider finitary logics (i.e. logics enjoying a Hilbert-style calculus where all rules have finitely-many premises). Therefore we set the following convention to delimit, in this paper (!), the class of substructural logics. You can check $[12,18]$ for other possible conventional definitions of this family of logics.

Convention 1. A logic is substructural if it is a finitary extension of SL.

\subsection{Algebraic semantics}

In this subsection we present the algebraic semantics for SL and other substructural logics. For this we need to recall several algebraic notions and fix some notation and terminology. ${ }^{4}$

A poset $\boldsymbol{P}=\langle P, \leq\rangle$ is a partially ordered set. If $\leq$ is a total order, then $\boldsymbol{P}$ is called a chain. A map $\gamma: P \rightarrow P$ is said to be a closure operator on $\boldsymbol{P}$ if it is expanding $(x \leq \gamma(x))$, monotone $(x \leq y$ implies $\gamma(x) \leq \gamma(y))$ and idempotent $(\gamma(\gamma(x))=\gamma(x))$. Dually, a map $\sigma: P \rightarrow P$ is called an interior operator on $\boldsymbol{P}$ provided that it is contracting $(\sigma(x) \leq x)$, monotone and idempotent. The elements in the image $\gamma[P]$ (resp. $\sigma[P])$ are called $\gamma$-closed (resp. $\sigma$-open).

Let $\boldsymbol{P}$ be a poset. A map $f: P \rightarrow P$ is residuated if there is a map $f^{\dagger}: P \rightarrow P$ such that for all $x, y \in P$ we have $f(x) \leq y$ iff $x \leq f^{\dagger}(y)$. Equivalently, $f$ is residuated iff $f$ is monotone and the inverse image of any principal downset is a principal downset as well (recall that a principal downset is a subset of $P$ of the form $\{y \in P \mid y \leq x\}$ for some $x \in P$ ). A binary operation $\circ: P^{2} \rightarrow P$ is residuated if it is residuated component-wise, i.e. for every $a \in P$ the maps given by $x \mapsto a \circ x$ and $x \mapsto x \circ a$ are residuated. Equivalently, there are maps $\backslash: P^{2} \rightarrow P$ and $/: P^{2} \rightarrow P$ such that for all $a, b, c \in P$ we have

$$
a \circ b \leq c \quad \text { iff } \quad b \leq a \backslash c \quad \text { iff } \quad a \leq c / b .
$$

The maps $\backslash, /$ are called respectively left and right residual of o.

A lattice is a poset where every pair of elements $x, y$ has a greatest lower bound $x \wedge y$ and the least upper bound $x \vee y$. A lattice $\boldsymbol{A}$ is called bounded provided that it has a minimum $\perp$ and a maximum $\top$. We call an algebra $\boldsymbol{A}=\langle A, \wedge, \vee, 0,1, \perp, \top\rangle$ a doubly pointed bounded lattice (shortly $d p b$-lattice) if $\langle A, \wedge, \vee, \perp, \top\rangle$ is a bounded lattice endowed with additional constants 0,1 . Let $\mathrm{T} \subseteq\{\mathrm{i}, \mathrm{o}\}$. Then a $d p b$-lattice is said to be a $d p b_{\mathrm{T}}$-lattice provided that $1=\top$ if $\mathrm{i} \in \mathrm{T}$ and $0=\perp$ if $\mathrm{o} \in \mathrm{T}$. We apply the same convention also for chains, i.e., a $d p b_{\mathrm{T}}$-chain is a chain which is also a $d p b_{\mathrm{T}}$-lattice.

\footnotetext{
${ }^{4}$ For unexplained notions, notations, and terminology of Universal Algebra used in the paper see e.g. [5].
} 
An algebra $\boldsymbol{A}=\langle A, \wedge, \vee, \cdot, \backslash, /, 0,1, \perp, \top\rangle$ is called (semiunital) residuated lattice ordered groupoid (shortly $r \ell$-groupoid) if $\langle A, \wedge, \vee, 0,1, \perp, \top\rangle$ is a $d p b$-lattice satisfying $x \leq(1 \cdot x) \wedge(x \cdot 1)$, the groupoid operation - is residuated, and its residuals are the operations $\backslash$ and $/$. Let $S \subseteq\{\mathrm{e}, \mathrm{c}, \mathrm{i}, \mathrm{o}\}$. An $r$-groupoid $\boldsymbol{A}=\langle A, \wedge, \vee, \cdot, \backslash, /, 0,1, \perp, \top\rangle$ is said to be an $r \ell_{\mathrm{S}}$-groupoid provided that

- if e $\in S$, then $x \cdot y=y \cdot x$ for all $x, y \in A,{ }^{5}$

- if $\mathrm{c} \in S$, then $x \leq x \cdot x$ for all $x \in A$,

- $\langle A, \wedge, \vee, 0,1, \perp, T\rangle$ is a $d p b_{\mathrm{T}}$-lattice for $\mathrm{T}=\mathrm{S} \backslash\{\mathrm{e}, \mathrm{c}\}$.

Definition 2. Let $\mathrm{S} \subseteq\{\mathrm{e}, \mathrm{c}, \mathrm{i}, \mathrm{o}\}$. An $r \ell_{\mathrm{S}}$-groupoid $\boldsymbol{A}=\langle A, \wedge, \vee, \cdot, \backslash, /, 0,1, \perp, \top\rangle$ is

- totally ordered if $\langle A, \wedge, \vee\rangle$ forms a chain (we also use the term rt $_{\mathrm{S}}$-groupoid, for short).

- unital if 1 is a neutral element for the groupoid operation, i.e., $1 \cdot x=x=x \cdot 1$.

Unital (totally ordered) $r \ell_{\mathrm{S}}-$ groupoids are also called $\mathrm{SL}_{\mathrm{S}}$-algebras (resp. $\mathrm{SL}_{\mathrm{S}}$-chains).

For $S=\emptyset$ we speak about SL-algebras and SL-chains. Observe that the residuation condition together with the fact that 1 is a neutral element implies that for every SL-algebra $\boldsymbol{A}$ and each $a, b \in A$ we have

$$
a \leq b \quad \text { iff } \quad 1 \leq a \backslash b \quad \text { iff } \quad 1 \leq b / a .
$$

Given an SL-algebra $\boldsymbol{A}=\langle A, \wedge, \vee, \cdot, \backslash, /, 0,1, \perp, \top\rangle$ an $\boldsymbol{A}$-evaluation is an homomorphism from the algebra of formulae to $\boldsymbol{A}$ such that the connectives $\wedge, \vee, \&, \rightarrow, \rightsquigarrow, \overline{0}, \overline{1}, \perp, \top$ are respectively interpreted by the functions $\wedge, \vee, \cdot, \backslash, /, 0,1, \perp, \top$. By means of this notion, we can give, more generally, the following definition for the algebraic counterpart of any substructural logic, which can easily be seen to encompass the previous cases.

Definition 3. Let $\mathrm{L}$ be the substructural logic obtained by adding a set of axioms $A X$ and a set of rules $R$ to SL. $\boldsymbol{A}=\langle A, \wedge, \vee, \cdot, \backslash, /, 0,1, \perp, \top\rangle$ is an L-algebra if it is an SL-algebra such that:

- for every $\varphi \in A X$ and every $\boldsymbol{A}$-evaluation $e, e(\varphi) \geq 1$,

- for every $\Gamma \vdash \varphi \in R$ and every $\boldsymbol{A}$-evaluation $e$, if $e(\psi) \geq 1$ for every $\psi \in \Gamma$, then $e(\varphi) \geq 1$.

The class of all SL-algebras, denoted as $\mathbb{S L}$, is well-known to be a variety and it gives a semantics for the logic SL. In general, for every substructural logic L the class $\mathbb{L}$ of L-algebras (clearly, a subquasivariety of $\mathbb{S L}$ ) gives a semantics for L. To formulate the corresponding completeness theorems, we need to define a notion of semantical consequence. Given a class $\mathbb{K} \subseteq \mathbb{S L}$, a set of formulae $\Gamma$ and a formula $\varphi, \Gamma \models_{\mathbb{K}} \varphi$ if for every $\boldsymbol{A} \in \mathbb{K}$ and every $\boldsymbol{A}$-evaluation $e$, if $e(\psi) \geq 1$ for every $\psi \in \Gamma$, then $e(\varphi) \geq 1$.

Theorem 4. Let L be a substructural logic. Then for every set of formulae $\Gamma$ and every formula $\varphi$ we have: $\Gamma \vdash_{\mathrm{L}} \varphi$ if, and only if, $\Gamma \models_{\mathbb{L}} \varphi$.

Technically speaking, SL is an algebraizable logic in the sense of [3] and $\mathbb{S L}$ is its equivalent algebraic semantics with translations $E(p, q)=\{p \rightarrow q, q \rightarrow p\}$ and $\mathcal{E}(p)=\{p \wedge \overline{1} \approx \overline{1}\}$. The same holds for every substructural logic $L$ and its corresponding quasivariety $\mathbb{L}$.

Given a substructural logic $\mathrm{L}$ and an $\mathcal{L}_{\mathrm{SL}}$-algebra $\boldsymbol{A}$, a set $F \subseteq A$ is an L-filter if for every set of formulae $\Gamma$ and every formula $\varphi$ such that $\Gamma \vdash_{\mathrm{L}} \varphi$ and every $\boldsymbol{A}$-evaluation $e$ it holds: if $e[\Gamma] \subseteq F$, then $e(\varphi) \in F$. By $\mathcal{F} i_{\mathrm{L}}(\boldsymbol{A})$ we denote the set of all L-filters over $\boldsymbol{A}$. Since $\mathcal{F} i_{\mathrm{L}}(\boldsymbol{A})$ is a closure system (it clearly contains $A$ and is closed under arbitrary intersections), one can define a notion of generated filter. Given $X \subseteq A$, the L-filter generated by $X$, denoted as $\operatorname{Fi}_{\mathrm{L}}^{\boldsymbol{A}}(X)$ is the least L-filter containing $X$ (we omit the indexes when clear from the context).

We will need the following generic characterization for membership in the filter generated by a set (later we will show more usual algebraic descriptions of filters).

\footnotetext{
${ }^{5}$ Note that in this case the residuals coincide and we so we can denote them both by $\rightarrow$.
} 
Proposition 5. Let $\mathrm{L}$ be the substructural logic obtained by adding a set of axioms $A X$ and a set of rules $R$ to SL. Furthermore let $\boldsymbol{A}$ be an L-algebra and $X \cup\{a\} \subseteq A$. Let us define sets $V_{A X} \subseteq A$ and $V_{R} \subseteq \mathcal{P}(A) \times A$ as $\{e(\psi) \mid e$ is an $\boldsymbol{A}$-evaluation and $\psi \in \bar{A} X\}$ and $\{\langle e[\Gamma], e(\psi)\rangle \mid$ $e$ is an $\boldsymbol{A}$-evaluation and $\Gamma \vdash \psi \in R\} .{ }^{6}$ Then $a \in \mathrm{Fi}_{\mathrm{L}}^{\boldsymbol{A}}(X)$ iff there is a finite sequence $\left\langle a_{0}, \ldots, a_{n}\right\rangle$ (called proof of $a$ from $X$ ) of elements of $A$ such that

- $a_{n}=a$,

- for every $i \leq n$, either $a_{i} \in X \cup V_{A X}$ or there is a non-empty $Z \subseteq\left\{a_{0}, \ldots, a_{i-1}\right\}$ such that $\left\langle Z, a_{i}\right\rangle \in V_{R}$

Algebraizability gives a correspondence between filters and (relative) congruences in L-algebras. Let $\boldsymbol{C o n}_{\mathbb{L}}(\boldsymbol{A})$ denote the lattice of congruences of $\boldsymbol{A}$ relative to $\mathbb{L}$, i.e. giving a quotient in $\mathbb{L}$. If $\mathbb{L}$ is a variety, then $\boldsymbol{C o n}_{\mathbb{L}}(\boldsymbol{A})$ contains all congruences of $\boldsymbol{A}$. The Leibniz operator $\Omega_{\boldsymbol{A}}$ is defined, for any $F \in \mathcal{F} i_{\mathrm{L}}(\boldsymbol{A})$, as $\Omega_{\boldsymbol{A}}(F)=\left\{\langle a, b\rangle \in A^{2} \mid a \backslash b \in F\right.$ and $\left.b \backslash a \in F\right\}$.

Proposition 6. Let $\mathrm{L}$ be a substructural logic and $\boldsymbol{A}$ an L-algebra. The Leibniz operator $\Omega_{\boldsymbol{A}}$ is a lattice isomorphism from $\mathcal{F} i_{\mathrm{L}}(\boldsymbol{A})$ to $\boldsymbol{C o n}_{\mathbb{L}}(\boldsymbol{A})$. Its inverse is the function that maps any $\theta \in \boldsymbol{C o n}_{\mathbb{L}}(\boldsymbol{A})$ to the filter $\{a \in A \mid\langle a \wedge 1,1\rangle \in \theta\}$.

Observe that the minimum filter is the one generated by the emptyset, $\operatorname{Fi}(\emptyset)$, and it must correspond to the identity congruence $I d_{\boldsymbol{A}}$. Therefore, using the previous proposition, we obtain that, on any L-algebra $\boldsymbol{A}, \operatorname{Fi}(\emptyset)=\{a \in A \mid a \geq 1\}$. This set is, of course, contained in any other filter.

Given a class of algebras $\mathbb{K}$ a non-trivial algebra $\boldsymbol{A}$ is (finitely) subdirectly irreducible relative to $\mathbb{K}$ if for every (finite non-empty) subdirect representation $\alpha$ of $\boldsymbol{A}$ with a family $\left\{\boldsymbol{A}_{i} \mid i \in I\right\} \subseteq \mathbb{K}$ there is $i \in I$ such that $\pi_{i} \circ \alpha$ is an isomorphism. The class of all (finitely) subdirectly irreducible algebras relative to $\mathbb{K}$ is denoted as $\mathbb{K}_{\mathrm{R}(\mathrm{F}) \mathrm{SI}}$. Of course $\mathbb{K}_{\mathrm{RSI}} \subseteq \mathbb{K}_{\mathrm{RFSI}}$.

Note that, since all SL-algebras have a lattice reduct and there exists a majority term for lattices, $\mathbb{S L}$ is a congruence distributive variety. Now consider any substructural logic L such that $\mathbb{L}$ is a variety (equivalently: $\mathrm{L}$ is an axiomatic extension of $\mathrm{SL}$ ); then relative congruences and congruences are the same and, by Proposition $6, \mathcal{F} i_{\mathrm{L}}(\boldsymbol{A})$ forms a distributive lattice, i.e. $\mathrm{L}$ is a filter-distributive logic. If $\mathbb{L}$ is a proper quasivariety, we cannot conclude that $\mathrm{L}$ is filterdistributive, because in this case the relative congruences do not form a sublattice of the congruence lattice (as proved in [30]).

\section{Almost (MP)-based non-associative substructural logics}

In this section we present our new general results on non-associative substructural logics. They are based on the notion of almost (MP)-based logic, which has been firstly introduced and studied in [12]. Before we recall this notion, we need to introduce same technical notions. Let $\operatorname{Var}$ be the fixed set of propositional variables in which we are writing the formulae of the language $\mathcal{L}_{\mathrm{SL}}$ and $\star$ be a new symbol, which acts as placeholder for a special kind of substitutions. A $\star$-formula is built using variables $\operatorname{Var} \cup\{\star\}$ and a $\star$-substitution is a substitution in the extended language. Let $\varphi$ be a $\star$-formula, $\delta$ be a $\star$-formula, and $\sigma$ a $\star$-substitution defined as $\sigma(\star)=\varphi$ and $\sigma p=p$ for $p \in \operatorname{Var}$. By $\delta(\varphi)$ we denote the $\star$-formula $\sigma \delta$; note that if $\varphi$ is a formula in the original set of variables, so is $\delta(\varphi)$.

Definition 7. Given a set of $\star$-formulae $\Gamma$, we define the set $\Gamma^{*}$ of $\star$-formulae as the smallest set such that

• $\star \in \Gamma^{*}$ and

- $\delta(\gamma) \in \Gamma^{*}$ for each $\delta \in \Gamma$ and each $\gamma \in \Gamma^{*}$.

\footnotetext{
${ }^{6}$ Note that if $\boldsymbol{A}=\boldsymbol{F} \boldsymbol{m}_{\mathcal{L}}$, then $V_{A X}=A X$ and $V_{R}=R$.
} 


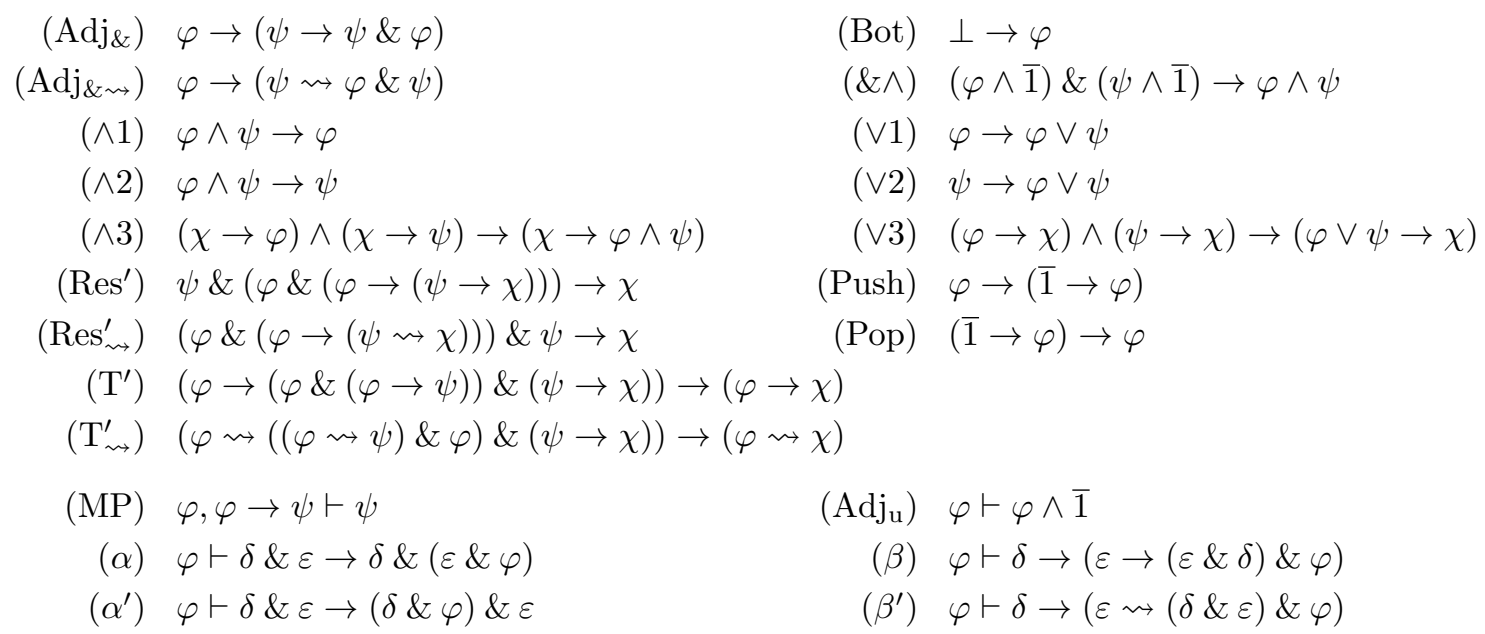

Table 3: New axiomatic system for SL

We are ready now to give the formal definition of almost (MP)-based logic.

Definition 8. Let bDT be a set of $\star$-formulae. A substructural logic $\mathrm{L}$ is almost (MP)-based w.r.t. the set of basic deduction terms bDT if:

- the set $\mathrm{bDT}$ is closed under all $\star$-substitutions $\sigma$ such that $\sigma(\star)=\star$,

- L has a presentation where the only deduction rules are modus ponens and those from $\{\varphi \vdash$ $\left.\gamma(\varphi) \mid \varphi \in F m_{\mathcal{L}_{\mathrm{SL}}}, \gamma \in \mathrm{bDT}\right\}$, and

- for each $\beta \in \mathrm{bDT}$ and each formulae $\varphi, \psi$, there exist $\beta_{1}, \beta_{2} \in \mathrm{bDT}^{*}$ such that: ${ }^{7}$

$$
\vdash_{\mathrm{L}} \beta_{1}(\varphi \rightarrow \psi) \rightarrow\left(\beta_{2}(\varphi) \rightarrow \beta(\psi)\right) .
$$

$\mathrm{L}$ is called (MP)-based if it admits the empty set as a set of basic deduction terms.

The goal of the first subsection is to obtain an equivalent Hilbert-style presentation of SL showing that this logic and thus its axiomatic extensions are indeed almost (MP)-based. In the second subsection we use this result to obtain local deduction theorems and a descriptions of generated filters in non-associative substructural logics. Finally, in the third subsection the terms appearing in the unary rules of almost (MP)-based presentations are used to build a generalized disjunction connective satisfying the Proof by Cases Property. Using this and following general results in [10], we obtain other logical and algebraic properties.

\subsection{Almost (MP)-based presentations of prominent substructural logics}

We start by providing an alternative system for SL.

Theorem 9. The axiomatic system from Table 3 is a presentation of SL.

Proof. We only have to provide formal proofs of all axioms and rules of one system in the other one and vice versa. The proofs are rather tedious and can be found in Appendix A.

\footnotetext{
${ }^{7}$ Here we are deviating from the original definition from [12] where $\beta_{1}, \beta_{2}$ were required to be in bDT. This alteration has no effect on the notion of almost (MP)-based logic as shown by claim 2 in Lemma 16 which can be read as: if bDT is a set of basic deduction terms in the sense just defined, then bDT* is a set of basic deduction terms in the original sense, so the logic remains almost (MP)-based. This new definition of bDT will however allow us to obtain stronger results in Subsection 4.1.
} 
Next we show that SL is an almost (MP)-based logic, first to introduce a convenient notation for the terms appearing on the right-hand side of the rules $(\alpha),\left(\alpha^{\prime}\right),(\beta)$, and $\left(\beta^{\prime}\right)$. Given arbitrary formulae $\delta, \varepsilon$, we define the following $\star$-formulae:

$$
\begin{array}{ll}
\alpha_{\delta, \varepsilon}=\delta \& \varepsilon \rightarrow \delta \&(\varepsilon \& \star) & \beta_{\delta, \varepsilon}=\delta \rightarrow(\varepsilon \rightarrow(\varepsilon \& \delta) \& \star) \\
\alpha_{\delta, \varepsilon}^{\prime}=\delta \& \varepsilon \rightarrow(\delta \& \star) \& \varepsilon & \beta_{\delta, \varepsilon}^{\prime}=\delta \rightarrow(\varepsilon \rightsquigarrow(\delta \& \varepsilon) \& \star)
\end{array}
$$

Note that these terms (those in the second line) generalize the well-known notions of left and right conjugates used in associative logics: ${ }^{8}$

$$
\lambda_{\varepsilon}=\varepsilon \rightarrow \star \& \varepsilon \quad \rho_{\varepsilon}=\varepsilon \rightsquigarrow \varepsilon \& \star
$$

The next, not difficult to prove, proposition shows how these terms, and hence the axiomatic systems in which they appear, can be simplified in stronger substructural logics (e.g. in presence of exchange we can omit the prime version of the rules and associativity allows us to replace $\alpha$, $\alpha^{\prime}, \beta, \beta^{\prime}$ by the rules $\varphi \vdash \rho_{\varepsilon}(\varphi)$ and $\left.\varphi \vdash \lambda_{\varepsilon}(\varphi)\right)$.

Proposition 10. We have

1. $\vdash_{\mathrm{SL}} \gamma_{\overline{1}, \overline{1}}(\varphi) \leftrightarrow \varphi$ for each $\gamma \in\left\{\alpha, \alpha^{\prime}, \beta, \beta^{\prime}\right\}$

2. $\vdash_{\mathrm{SL}_{\mathrm{e}}} \alpha_{\delta, \varepsilon}(\varphi) \leftrightarrow \alpha_{\varepsilon, \delta}^{\prime}(\varphi)$ and $\vdash_{\mathrm{SL}_{\mathrm{e}}} \beta_{\delta, \varepsilon}(\varphi) \leftrightarrow \beta_{\delta, \varepsilon}^{\prime}(\varphi)$

3. $\vdash_{\mathrm{SL}_{\mathrm{a}}} \varphi \rightarrow \gamma_{\delta, \varepsilon}(\varphi)$ for each $\gamma \in\{\alpha, \beta\}$

4. $\vdash_{\mathrm{SL}_{\mathrm{a}}} \lambda_{\varepsilon}(\varphi) \rightarrow \alpha_{\delta, \varepsilon}^{\prime}(\varphi)$ and $\vdash_{\mathrm{SL}_{\mathrm{a}}} \rho_{\varepsilon}(\varphi) \rightarrow \beta_{\delta, \varepsilon}^{\prime}(\varphi)$

5. $\vdash_{\mathrm{SL}_{\mathrm{a}}} \lambda_{\varepsilon}(\varphi) \leftrightarrow \alpha_{\overline{1}, \varepsilon}^{\prime}(\varphi)$ and $\vdash_{\mathrm{SL}_{\mathrm{a}}} \rho_{\varepsilon}(\varphi) \leftrightarrow \beta_{\overline{1}, \varepsilon}^{\prime}(\varphi)$

6. $\vdash_{\mathrm{SL}_{\mathrm{ae}}} \varphi \rightarrow \lambda_{\varepsilon}(\varphi)$ and $\vdash_{\mathrm{SL}_{\mathrm{ae}}} \varphi \rightarrow \rho_{\varepsilon}(\varphi)$

Now, by means of two syntactical lemmata, we can obtain the main result of this section.

Lemma 11. The following are provable in SL:

$$
\begin{aligned}
& \text { (Aux1) } \vdash \alpha_{\chi, \varphi}(\varphi \rightarrow \psi) \rightarrow(\chi \& \varphi \rightarrow \chi \& \psi) \\
& \text { (Aux2) } \vdash \alpha_{\varphi, \chi}^{\prime}(\varphi \rightarrow \psi) \rightarrow(\varphi \& \chi \rightarrow \psi \& \chi) \\
& \left(\text { Aux3) } \vdash \beta_{\chi \rightarrow \varphi, \chi}(\varphi \rightarrow \psi) \rightarrow((\chi \rightarrow \varphi) \rightarrow(\chi \rightarrow \psi))\right. \\
& (\text { Aux4 }) \vdash \beta_{\chi \rightsquigarrow \varphi, \chi}^{\prime}(\varphi \rightarrow \psi) \rightarrow((\chi \rightsquigarrow \varphi) \rightarrow(\chi \rightsquigarrow \psi))
\end{aligned}
$$

Proof. - SL proves (Aux1):

(a) $\vdash \varphi \&(\varphi \rightarrow \psi) \rightarrow \psi$

(b) $\vdash \chi \&(\varphi \&(\varphi \rightarrow \psi)) \rightarrow \chi \& \psi$

(c) $\vdash(\chi \& \varphi \rightarrow \chi \&(\varphi \&(\varphi \rightarrow \psi))) \rightarrow(\chi \& \varphi \rightarrow \chi \& \psi)$

(b) and (Pf)

- SL proves (Aux2):
(a) $\vdash \varphi \&(\varphi \rightarrow \psi) \rightarrow \psi$
(b) $\vdash(\varphi \&(\varphi \rightarrow \psi)) \& \chi \rightarrow \psi \& \chi$
(c) $\vdash(\varphi \& \chi \rightarrow(\varphi \&(\varphi \rightarrow \psi)) \& \chi) \rightarrow(\varphi \& \chi \rightarrow \psi \& \chi)$

(a) and $\left(\mathrm{P}_{\mathrm{SL}} 9\right)$

(b) and (Pf)

\footnotetext{
${ }^{8}$ It is usual in the literature on algebraic study of substructural logics to find these terms defined in a slightly more complicated way: $\lambda_{\varepsilon}=(\varepsilon \rightarrow \star \& \varepsilon) \wedge \overline{1}$ and $\rho_{\varepsilon}=(\varepsilon \rightsquigarrow \varepsilon \& \star) \wedge \overline{1}$, although in the usual Hilbert-style axiomatizations of FL the simplified terms without $\wedge \overline{1}$ are used for the product normality rules. The reason for this more complicated form is to give algebraic terms which simultaneously cope with product normality rules and adjunction, whereas our formalism allows for a clearer distinction of their respective rôles.
} 
- SL proves (Aux3):
(a) $\vdash \chi \&(\chi \rightarrow \varphi) \rightarrow \varphi$
$\left(\mathrm{P}_{\mathrm{SL}} 2\right)$
(b) $\vdash(\chi \&(\chi \rightarrow \varphi)) \&(\varphi \rightarrow \psi) \rightarrow \varphi \&(\varphi \rightarrow \psi)$
(a) and $\left(\mathrm{P}_{\mathrm{SL}} 9\right)$
(c) $\vdash \varphi \&(\varphi \rightarrow \psi) \rightarrow \psi$
(d) $\vdash(\chi \&(\chi \rightarrow \varphi)) \&(\varphi \rightarrow \psi) \rightarrow \psi$
(b), (c), and (T)
(e) $\vdash(\chi \rightarrow(\chi \&(\chi \rightarrow \varphi)) \&(\varphi \rightarrow \psi)) \rightarrow(\chi \rightarrow \psi)$
(d) and $(\mathrm{Pf})$
(f) $\quad \vdash[(\chi \rightarrow \varphi) \rightarrow(\chi \rightarrow(\chi \&(\chi \rightarrow \varphi)) \&(\varphi \rightarrow \psi))] \rightarrow[(\chi \rightarrow \varphi) \rightarrow(\chi \rightarrow \psi)]$
(e) and (Pf)

- SL proves $(\operatorname{Aux} 4)$ :
(a) $\vdash(\chi \rightsquigarrow \varphi) \& \chi \rightarrow \varphi$
$\left(\mathrm{As}_{\ell \ell}\right)$ and $\left(\operatorname{Res}_{1}\right)$
(b) $\vdash((\chi \rightsquigarrow \varphi) \& \chi) \&(\varphi \rightarrow \psi) \rightarrow \varphi \&(\varphi \rightarrow \psi)$
(a) and $\left(\mathrm{P}_{\mathrm{SL}} 9\right)$
(c) $\vdash \varphi \&(\varphi \rightarrow \psi) \rightarrow \psi$
(d) $\vdash((\chi \rightsquigarrow \varphi) \& \chi) \&(\varphi \rightarrow \psi) \rightarrow \psi$
(b), (c), and (T)
(e) $\vdash(\chi \rightsquigarrow((\chi \rightsquigarrow \varphi) \& \chi) \&(\varphi \rightarrow \psi)) \rightarrow(\chi \rightsquigarrow \psi)$
(d) and $(\mathrm{Pf})_{\rightsquigarrow}$
(f) $\quad \vdash[(\chi \rightsquigarrow \varphi) \rightarrow(\chi \rightsquigarrow((\chi \rightsquigarrow \varphi) \& \chi) \&(\varphi \rightarrow \psi))] \rightarrow[(\chi \rightsquigarrow \varphi) \rightarrow(\chi \rightsquigarrow \psi)]$ (e) and (Pf)

Lemma 12. For every $\star$-formula $\gamma \in\left\{\alpha_{\delta, \varepsilon}, \alpha_{\delta, \varepsilon}^{\prime}, \beta_{\delta, \varepsilon}, \beta_{\delta, \varepsilon}^{\prime} \mid \delta, \varepsilon\right.$ formulae $\}$ and every pair of formulae $\varphi, \psi$, we have: $\varphi \rightarrow \psi \vdash_{\mathrm{SL}} \gamma(\varphi) \rightarrow \gamma(\psi)$.

Proof. All the cases are easily proved in a similar way. Let us show the case of $\alpha_{\delta, \varepsilon}$ as an example.

(a) $\varphi \rightarrow \psi \vdash \delta \&(\varepsilon \& \varphi) \rightarrow \delta \&(\varepsilon \& \psi)$

$\left(\mathrm{P}_{\mathrm{SL}} 8\right)$ twice

(b) $\varphi \rightarrow \psi \vdash(\delta \& \varepsilon \rightarrow \delta \&(\varepsilon \& \varphi)) \rightarrow(\delta \& \varepsilon \rightarrow \delta \&(\varepsilon \& \psi))$

(a) and (Pf)

Theorem 13. SL is almost (MP)-based with respect to the set

$$
\mathrm{bDT}_{\mathrm{SL}}=\left\{\alpha_{\delta, \varepsilon}, \alpha_{\delta, \varepsilon}^{\prime}, \beta_{\delta, \varepsilon}, \beta_{\delta, \varepsilon}^{\prime}, \star \wedge \overline{1} \mid \delta, \varepsilon \text { formulae }\right\} .
$$

Proof. Theorem 9 shows that there is a presentation of SL with (MP) as the only binary rule and unary rules $\varphi \vdash \gamma(\varphi)$ for each $\gamma \in \mathrm{bDT}_{\mathrm{SL}}$. We need to prove the final condition in the definition of almost (MP)-based axiomatic systems, in particular we show that for each $\gamma \in \mathrm{bDT}_{\mathrm{SL}}$ and each formulae $\varphi, \psi$ there is $\gamma^{\prime} \in \mathrm{bDT}_{\mathrm{SL}}^{*}$ such that

$$
\vdash \gamma^{\prime}(\varphi \rightarrow \psi) \rightarrow(\gamma(\varphi) \rightarrow \gamma(\psi)) .
$$

If $\gamma$ is $\star \wedge \overline{1}$ we can set $\gamma^{\prime}=\gamma$ due to $\left(\mathrm{P}_{\mathrm{SL}} 24\right)$. Next we prove the claim for $\alpha_{\delta, \varepsilon}^{\prime}$, the other cases are proved analogously:

(a) $\alpha_{\delta, \varphi}(\varphi \rightarrow \psi) \rightarrow[\delta \& \varphi \rightarrow \delta \& \psi]$

(b) $\alpha_{\delta \& \varphi, \varepsilon}^{\prime}(\delta \& \varphi \rightarrow \delta \& \psi) \rightarrow[(\delta \& \varphi) \& \varepsilon \rightarrow(\delta \& \psi) \& \varepsilon]$

(c) $\beta_{\delta \& \varepsilon \rightarrow(\delta \& \varphi) \& \varepsilon, \delta \& \varepsilon}((\delta \& \varphi) \& \varepsilon \rightarrow(\delta \& \psi) \& \varepsilon) \rightarrow\left[\alpha_{\delta, \varepsilon}^{\prime}(\varphi) \rightarrow \alpha_{\delta, \varepsilon}^{\prime}(\psi)\right]$

(d) $\quad \alpha_{\delta \& \varphi, \varepsilon}^{\prime}\left(\alpha_{\delta, \varphi}(\varphi \rightarrow \psi)\right) \rightarrow \alpha_{\delta \& \varphi, \varepsilon}^{\prime}(\delta \& \varphi \rightarrow \delta \& \psi)$

(e) $\quad \alpha_{\delta \& \varphi, \varepsilon}^{\prime}\left(\alpha_{\delta, \varphi}(\varphi \rightarrow \psi)\right) \rightarrow[(\delta \& \varphi) \& \varepsilon \rightarrow(\delta \& \psi) \& \varepsilon]$

(a) and Lemma 12

(f) $\quad \beta_{\delta \& \varepsilon \rightarrow(\delta \& \varphi) \& \varepsilon, \delta \& \varepsilon}\left(\alpha_{\delta \& \varphi, \varepsilon}^{\prime}\left(\alpha_{\delta, \varphi}(\varphi \rightarrow \psi)\right)\right) \rightarrow\left[\alpha_{\delta, \varepsilon}^{\prime}(\varphi) \rightarrow \alpha_{\delta, \varepsilon}^{\prime}(\psi)\right]$

(e), Lemma 12, and (c)

At the end of this subsection we show how we can simplify the sets of basic deductive terms in prominent axiomatic extensions of SL. The results are summarized in Table 4; in the case of $\mathrm{SL}_{\mathrm{e}}$ it follows from the second claim of Proposition 10, in case of logics with weakening we use the fact that the rule $\left(\operatorname{Adj}_{\mathrm{u}}\right)$ is redundant and the term $\star \wedge \overline{1}$ is not needed in the crucial step of the proof in Theorem 13; for associative logics it implicitly follows from [12, Theorem 2.6.8], or from the following result which we add for the reader's convenience. 


\begin{tabular}{|l|l|}
\hline Logic $\mathrm{L}$ & $\mathrm{bDT}_{\mathrm{L}}$ \\
\hline \hline $\mathrm{SL}$ & $\left\{\alpha_{\delta, \varepsilon}, \alpha_{\delta, \varepsilon}^{\prime}, \beta_{\delta, \varepsilon}, \beta_{\delta, \varepsilon}^{\prime}, \star \wedge \overline{1} \mid \delta, \varepsilon\right.$ formulae $\}$ \\
\hline $\mathrm{SL}_{\mathrm{w}}$ & $\left\{\alpha_{\delta, \varepsilon}, \alpha_{\delta, \varepsilon}^{\prime}, \beta_{\delta, \varepsilon}, \beta_{\delta, \varepsilon}^{\prime} \mid \delta, \varepsilon\right.$ formulae $\}$ \\
\hline $\mathrm{SL}_{\mathrm{e}}$ & $\left\{\alpha_{\delta, \varepsilon}, \beta_{\delta, \varepsilon}, \star \wedge \overline{1} \mid \delta, \varepsilon\right.$ formulae $\}$ \\
\hline $\mathrm{SL}_{\mathrm{ew}}$ & $\left\{\alpha_{\delta, \varepsilon}, \beta_{\delta, \varepsilon} \mid \delta, \varepsilon\right.$ formulae $\}$ \\
\hline $\mathrm{SL}_{\mathrm{a}}$ & $\left\{\lambda_{\varepsilon}, \rho_{\varepsilon}, \star \wedge \overline{1} \mid \varepsilon\right.$ a formula $\}$ \\
\hline $\mathrm{SL}_{\mathrm{ae}}$ & $\{\star \wedge \overline{1}\}$ \\
\hline $\mathrm{SL}_{\mathrm{aew}}$ & $\{\star\}$ \\
\hline
\end{tabular}

Table 4: bDTs of prominent substructural logics

Corollary 14. $\mathrm{SL}_{\mathrm{a}}$ is almost (MP)-based with respect to the set

$$
\mathrm{bDT}_{\mathrm{SL}_{\mathrm{a}}}=\left\{\lambda_{\varepsilon}, \rho_{\varepsilon}, \star \wedge \overline{1} \mid \varepsilon \text { a formula }\right\} .
$$

Proof. The fact that $\mathrm{SL}_{\mathrm{a}}$ can be axiomatized by using the rules $\varphi \vdash \gamma(\varphi)$ for $\gamma \in \mathrm{bDT}_{\mathrm{SL}_{\mathrm{a}}}$ follows from claims 3, 4, and 5 of Proposition 10.

From the proof of the previous theorem and claim 5 of Proposition 10 we know that for each $\gamma \in \mathrm{bDT}_{\mathrm{SL}_{\mathrm{a}}}$ and each formulae $\varphi, \psi$ there is $\gamma^{\prime} \in \mathrm{bDT}_{\mathrm{SL}}^{*}$ such that

$$
\vdash_{\mathrm{SL}} \gamma^{\prime}(\varphi \rightarrow \psi) \rightarrow(\gamma(\varphi) \rightarrow \gamma(\psi))
$$

We complete the proof by showing that for each $\gamma^{\prime} \in \mathrm{bDT}_{\mathrm{SL}}^{*}$ there is $\gamma_{0} \in \mathrm{bDT}_{\mathrm{SL}_{\mathrm{a}}}^{*}$ such that for each formula $\chi$ holds:

$$
\vdash_{\mathrm{SL}_{\mathrm{a}}} \gamma_{0}(\chi) \rightarrow \gamma^{\prime}(\chi)
$$

The base case follows from claims 1, 3, and 4 of Proposition 10. The induction step then easily follows using Lemma 12 and claim 3 and 4 again.

\subsection{Deduction theorem and filter generation}

In this section we prove a general form of (parameterized) local deduction theorem for almost (MP)-based substructural logics and use it to obtain a description of generated filters. To this end, we need first a few additional syntactical properties of sets of (iterated) basic deduction terms and their closures under conjunction.

Definition 15. Given a set of $\star$-formulae $\Gamma$, an SL-algebra $\boldsymbol{A}$, and a set $X \subseteq A$, we define

- $\Pi(\Gamma)$ as the smallest set of $\star$-formulae containing $\Gamma \cup\{\overline{1}\}$ and closed under \&.

- $\Gamma^{\boldsymbol{A}}$ as the set of polynomials with coefficients from $A$ and variable $\star$, i.e., $\left\{\delta\left(\star, a_{1}, \ldots, a_{n}\right) \mid\right.$ $\delta\left(\star, p_{1}, \ldots, p_{n}\right) \in \Gamma$ and $\left.a_{1}, \ldots, a_{n} \in A\right\}$.

- $\Gamma^{\boldsymbol{A}}(X)$ as the set $\left\{\delta^{\boldsymbol{A}}(x) \mid \delta(\star) \in \Gamma^{\boldsymbol{A}}\right.$ and $\left.x \in X\right\}$.

We omit the symbol $\boldsymbol{A}$ when known from the context.

Lemma 16. Let L be a substructural logic and assume that it is almost (MP)-based with a set of basic deduction terms bDT. Then

1. for each $\gamma \in \mathrm{bDT}^{*}$ and formulae $\varphi, \psi$ there exists $\gamma^{\prime} \in \mathrm{bDT}^{*}$ such that

$$
\varphi \rightarrow \psi \vdash_{\mathrm{L}} \gamma^{\prime}(\varphi) \rightarrow \gamma(\psi)
$$


2. for each $\gamma \in \mathrm{bDT}^{*}$ and formulae $\varphi, \psi$ there exist $\gamma_{1}, \gamma_{2} \in \mathrm{bDT}^{*}$ such that

$$
\vdash_{\mathrm{L}} \gamma_{1}(\varphi \rightarrow \psi) \rightarrow\left(\gamma_{2}(\varphi) \rightarrow \gamma(\psi)\right)
$$

3. for each $\gamma \in \mathrm{bDT}^{*}$ and formulae $\varphi, \psi$ there exist $\gamma_{1}, \gamma_{2} \in \mathrm{bDT}^{*}$ such that

$$
\vdash_{\mathrm{L}} \gamma_{1}(\varphi) \& \gamma_{2}(\psi) \rightarrow \gamma(\varphi \& \psi)
$$

4. for each $\gamma \in \mathrm{bDT}^{*}, \delta \in \Pi\left(\mathrm{bDT}^{*}\right)$, and a formula $\varphi$ there exist $\hat{\delta} \in \Pi\left(\mathrm{bDT}^{*}\right)$ such that

$$
\vdash_{\mathrm{L}} \hat{\delta}(\varphi) \rightarrow \gamma(\delta(\varphi)) .
$$

Proof. We prove the first two claims at once by induction. The base case $\gamma=\star$ is trivial in both claims. Assume that $\gamma=\beta(\delta)$ for some $\beta \in \mathrm{bDT}$ and $\delta \in \mathrm{bDT}^{*}$. The induction assumption of the first claim gives us $\delta^{\prime} \in \mathrm{bDT}^{*}$ such that

$$
\varphi \rightarrow \psi \vdash_{\mathrm{L}} \delta^{\prime}(\varphi) \rightarrow \delta(\psi)
$$

Now we use the definition of bDT for $\delta^{\prime}(\varphi)$ and $\delta(\psi)$ and obtain $\beta_{1}, \beta_{2} \in \mathrm{bDT}^{*}$ such that:

$$
\vdash_{\mathrm{L}} \beta_{1}\left(\delta^{\prime}(\varphi) \rightarrow \delta(\psi)\right) \rightarrow\left(\beta_{2}\left(\delta^{\prime}(\varphi)\right) \rightarrow \beta(\delta(\psi))\right) .
$$

Thus if we set $\gamma^{\prime}=\beta_{2}\left(\delta^{\prime}\right)$ the proof of the first claim is done (just observe that $\varphi \rightarrow \psi \vdash_{\mathrm{L}}$ $\left.\beta_{1}\left(\delta^{\prime}(\varphi) \rightarrow \delta(\psi)\right)\right)$.

In the second claim, assuming again that $\gamma=\beta(\delta)$ for some $\beta \in \mathrm{bDT}$ and $\delta \in \mathrm{bDT}^{*}$, the induction assumption gives us $\delta_{1}, \delta_{2} \in \mathrm{bDT}^{*}$ such that

$$
\vdash_{\mathrm{L}} \delta_{1}(\varphi \rightarrow \psi) \rightarrow\left(\delta_{2}(\varphi) \rightarrow \delta(\psi)\right),
$$

Now we use the definition of bDT for $\delta_{2}(\varphi)$ and $\delta(\psi)$ and obtain $\beta_{1}, \beta_{2} \in \mathrm{bDT}^{*}$ such that:

$$
\vdash_{\mathrm{L}} \beta_{1}\left(\delta_{2}(\varphi) \rightarrow \delta(\psi)\right) \rightarrow\left(\beta_{2}\left(\delta_{2}(\varphi)\right) \rightarrow \beta(\delta(\psi))\right) .
$$

Now we apply the first claim for $\gamma=\beta_{1}, \varphi=\delta_{1}(\varphi \rightarrow \psi), \psi=\delta_{2}(\varphi) \rightarrow \delta(\psi)$ and obtain $\beta_{1}^{\prime} \in$ bDT $^{*}$ such that

$$
\vdash_{\mathrm{L}} \beta_{1}^{\prime}\left(\delta_{1}(\varphi \rightarrow \psi)\right) \rightarrow \beta_{1}\left(\delta_{2}(\varphi) \rightarrow \delta(\psi)\right) .
$$

Transitivity and setting $\gamma_{1}=\beta_{1}^{\prime}\left(\delta_{1}\right)$ and $\gamma_{2}=\beta_{2}\left(\delta_{2}\right)$ completes the proof of the second claim.

To prove the third claim we use the second one for $\psi=\varphi \& \psi$ and obtain $\gamma_{1}, \gamma_{2} \in \mathrm{bDT}^{*}$

$$
\vdash_{\mathrm{L}} \gamma_{1}(\varphi \rightarrow \varphi \& \psi) \rightarrow\left(\gamma_{2}(\varphi) \rightarrow \gamma(\varphi \& \psi)\right) .
$$

Since $\vdash_{\mathrm{L}} \psi \rightarrow(\varphi \rightarrow \varphi \& \psi)\left(\operatorname{Adj}_{\&}\right)$ we can use the first claim for $\gamma=\gamma_{1}$ to obtain $\gamma_{1}^{\prime} \in$ bDT $^{*}$ such that

$$
\vdash_{\mathrm{L}} \gamma_{1}^{\prime}(\psi) \rightarrow \gamma_{1}(\varphi \rightarrow \varphi \& \psi) .
$$

Claim 3 then simply follows by $(\mathrm{T})$ and $\left(\operatorname{Res}_{1}\right)$.

To prove the last claim we proceed by induction via the depth of the tree representing $\delta$. If $\delta \in \mathrm{bDT}^{*}$ or $\delta=\overline{1}$ the proof is done by setting $\hat{\delta}=\gamma(\delta)$ or $\hat{\delta}=\overline{1}$ respectively. Next assume that $\delta=\eta_{1} \& \eta_{2}$ for some $\eta_{1}, \eta_{2} \in \Pi\left(\mathrm{bDT}^{*}\right)$. By the third claim we obtain $\gamma_{1}, \gamma_{2} \in$ bDT $^{*}$ such that $\vdash_{\mathrm{L}} \gamma_{1}\left(\eta_{1}(\varphi)\right) \& \gamma_{2}\left(\eta_{2}(\varphi)\right) \rightarrow \gamma\left(\eta_{1}(\varphi) \& \eta_{2}(\varphi)\right)$. Then by the induction assumption we obtain $\hat{\delta}_{1}, \hat{\delta}_{2} \in \Pi\left(\mathrm{bDT}^{*}\right)$ such that $\vdash_{\mathrm{L}} \hat{\delta}_{1}(\varphi) \rightarrow \gamma_{1}\left(\eta_{1}(\varphi)\right)$ and $\vdash_{\mathrm{L}} \hat{\delta}_{2}(\varphi) \rightarrow \gamma_{2}\left(\eta_{2}(\varphi)\right)$. Setting $\hat{\delta}=\hat{\delta}_{1} \& \hat{\delta}_{2}$ completes the proof using $\left(\mathrm{P}_{\mathrm{SL}} 10\right)$.

We are ready now to prove a semantical (or transferred) version of (parameterized) local deduction theorem (cf. [12, Theorem 2.6.3]). 
Theorem 17. Let $\mathrm{L}$ be an almost (MP)-based substructural logic with a set of basic deduction

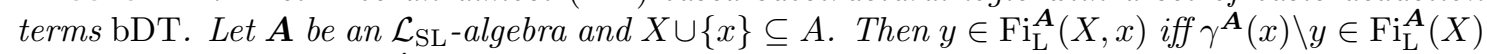
for some $\gamma \in\left(\Pi\left(\mathrm{bDT}^{*}\right)\right)^{\boldsymbol{A}}$.

Proof. Right-to-left direction: clearly $\gamma(x) \in \operatorname{Fi}(X, x)$ (because $\varphi \vdash \gamma_{0}(\varphi)$ for each $\gamma_{0} \in \mathrm{bDT}^{*}$, $\varphi, \psi \vdash \varphi \& \psi$ and $\operatorname{Fi}(X, x)$ is closed under the rules of $\mathrm{L})$. Since $\operatorname{Fi}(X, x)$ is closed under modus ponens we obtain that $y \in \operatorname{Fi}(X, x)$.

To prove the other direction let us take $y \in \operatorname{Fi}(X, x)$, we show that for each $a$ in a proof of $y$ from the assumptions $X \cup\{x\}$ (recall Proposition 5) there is $\gamma_{a} \in \Pi\left(\mathrm{bDT}^{*}\right)$ such that $\gamma_{a}(x) \backslash a \in \mathrm{Fi}(X)$. If $a=x$ we set $\gamma_{a}=\star$; if $a$ is in $X$ or is the value of some axiom we set $\gamma_{a}=\overline{1}$.

Assume that $a$ is obtained by modus ponens from $b \in \operatorname{Fi}(X, x)$ and $b \backslash a \in \operatorname{Fi}(X, x)$. By induction hypothesis, we have $\gamma_{b}, \gamma_{b \backslash a} \in \Pi\left(\mathrm{bDT}^{*}\right)$ such that $\gamma_{b}(x) \backslash b, \gamma_{b \backslash a}(x) \backslash(b \backslash a) \in \mathrm{Fi}(X)$. Therefore (using (Sf)) we have $(b \backslash a) \backslash\left(\gamma_{b}(x) \backslash a\right) \in \operatorname{Fi}(X)$ and by transitivity $\gamma_{b \backslash a}(x) \backslash\left(\gamma_{b}(x) \backslash a\right) \in \operatorname{Fi}(X)$. The proof is done by setting $\gamma_{a}=\gamma_{b} \cdot \gamma_{b \backslash a}$ and using residuation.

Assume that $a=\beta(b)$ from some $\beta \in \mathrm{bDT}$ and is obtained from $b \in \operatorname{Fi}(X, x)$ by the rule $\varphi \vdash \beta(\varphi)$. By the induction hypothesis, we have $\gamma_{b} \in \Pi\left(\mathrm{bDT}^{*}\right)$ such that $\gamma_{b}(x) \backslash b \in \operatorname{Fi}(X)$. Using the first claim of Lemma 16 we obtain $\gamma \in \mathrm{bDT}^{*}$ such that $\gamma\left(\gamma_{b}(x)\right) \backslash \beta(b) \in \mathrm{Fi}(X)$. Using the fourth claim of Lemma 16 we obtain $\hat{\gamma}_{b} \in \Pi\left(\mathrm{bDT}^{*}\right)$ such that $\hat{\gamma}_{b}(x) \backslash \gamma\left(\gamma_{b}(x)\right) \in \operatorname{Fi}(X)$ and so transitivity completes the proof.

This theorem has two important consequences; the first one is a straightforward corollary in the particular case when $\boldsymbol{A}$ is the algebra of formulae and recalling that in this case $\varphi \in \operatorname{Fi}(\Gamma)$ iff $\Gamma \vdash_{\mathrm{L}} \varphi$.

Corollary 18 (Local Deduction theorem). Let L be an almost (MP)-based substructural logic with a set of basic deduction terms bDT. Then for each set $\Gamma \cup\{\varphi, \psi\}$ of formulae the following holds:

$$
\Gamma, \varphi \vdash_{\mathrm{L}} \psi \quad \text { iff } \quad \Gamma \vdash_{\mathrm{L}} \gamma(\varphi) \rightarrow \psi \text { for some } \gamma \in \Pi\left(\mathrm{bDT}^{*}\right) \text {. }
$$

Therefore, we obtain a (parameterized or not-parameterized, depending on the presence of parameters in the set bDT) local deduction theorem for SL and its axiomatic extensions (sometimes with a simplified set bDT; see Table 4). On the other hand, Theorem 17 can be used to obtain the following algebraic description of the filter generated by a set.

Corollary 19 (Filter generation). Let L be an almost (MP)-based substructural logic with a set of basic deduction terms bDT. Let $\boldsymbol{A}$ be an L-algebra and $X \subseteq A$. Then $\operatorname{Fi}_{\mathrm{L}}^{\boldsymbol{A}}(X)=\{a \in A \mid a \geq x$ for some $\left.x \in\left(\Pi\left(\mathrm{bDT}^{*}\right)\right)^{\boldsymbol{A}}(X)\right\}$.

Proof. Clearly bDT $\mathrm{bD}^{*}(X) \subseteq \operatorname{Fi}(X)$ (because $\varphi \vdash \gamma(\varphi)$ for each $\gamma \in \mathrm{bDT}^{*}$ and $\mathrm{Fi}(X)$ is closed under the rules of L). Furthermore we obtain $\left(\Pi\left(\mathrm{bDT}^{*}\right)\right)^{\boldsymbol{A}}(X) \subseteq \operatorname{Fi}(X)$ from $\varphi, \psi \vdash \varphi \& \psi$. Finally take $x \in\left(\Pi\left(\mathrm{bDT}^{*}\right)\right)^{\boldsymbol{A}}(X)$. We know that $a \geq x$ implies that $x \backslash a \geq 1$ and so $x \backslash a \in \operatorname{Fi}(X)$. Thus the closedness of $\mathrm{Fi}(X)$ under modus ponens completes the proof of one direction.

To prove the other inclusion assume that $a \in \operatorname{Fi}(X)$. There has to be a finite set $\left\{x_{1}, \ldots x_{n}\right\}=$ $X^{\prime} \subseteq X$ such that $a \in \operatorname{Fi}\left(X^{\prime}\right)$ (due to Proposition 5). Repeated use of the previous theorem gives us $\gamma_{1}, \ldots, \gamma_{n} \in\left(\Pi\left(\mathrm{bDT}^{*}\right)\right)^{\boldsymbol{A}}$ such that

$$
\left.\gamma_{n}\left(x_{n}\right) \cdot\left(\ldots \cdot \gamma_{1}\left(x_{1}\right)\right) \ldots\right) \backslash a=\gamma_{1}\left(x_{1}\right) \backslash\left(\gamma_{2}\left(x_{2}\right) \backslash \ldots\left(\gamma_{n}\left(x_{n}\right) \backslash a\right) \ldots\right) \in \operatorname{Fi}(\emptyset)=\{x \mid x \geq 1\} .
$$

Therefore $a \geq x$ for $\left.x=\gamma_{n}\left(x_{n}\right) \cdot\left(\ldots \cdot \gamma_{1}\left(x_{1}\right)\right) \ldots\right) \in\left(\Pi\left(\mathrm{bDT}^{*}\right)\right)^{\boldsymbol{A}}(X)$.

\subsection{Proof by Cases Property and its applications}

In Abstract Algebraic Logic, the classical Proof by Cases Property:

$$
\frac{\Gamma, \varphi \vdash \chi \quad \Gamma, \psi \vdash \chi}{\Gamma, \varphi \vee \psi \vdash \chi}
$$


has inspired a systematical study of disjunction connectives, by means of a generalized form of the meta-rule which leads to a generalized notion of disjunction. Following the notation and terminology from [10], given an $\mathcal{L}_{\mathrm{SL}}$-algebra $\boldsymbol{A}$, sets $X, Y \subseteq A$, and a set of formulae $\nabla(p, q, \vec{r})$ in two variables $p, q$ and possibly parameters $\vec{r}$ we define

$$
X \nabla^{\boldsymbol{A}} Y=\left\{\delta^{\boldsymbol{A}}\left(x, y, a_{1}, \ldots, a_{n}\right) \mid \delta\left(p, q, p_{1}, \ldots, p_{n}\right) \in \nabla, x \in X, y \in Y, \text { and } a_{1}, \ldots, a_{n} \in A\right\} .
$$

Again, we omit the symbol $\boldsymbol{A}$ when known from the context. Finally, we set one more convention: we write $\Gamma \vdash \Delta$ instead of $\Gamma \vdash \psi$ for each $\psi \in \Delta$.

Definition 20. Given a logic L, a set of formulae $\nabla(p, q, \vec{r})$ is called a p-disjunction (in L) whenever it satisfies the p-protodisjunction condition

$$
\varphi \vdash_{\mathrm{L}} \varphi \nabla \psi \quad \text { and } \quad \psi \vdash_{\mathrm{L}} \varphi \nabla \psi \text {. }
$$

and the Proof by Cases Property, PCP for short:

$$
\frac{\Gamma, \varphi \vdash_{\mathrm{L}} \chi \quad \Gamma, \psi \vdash_{\mathrm{L}} \chi}{\Gamma, \varphi \nabla \psi \vdash_{\mathrm{L}} \chi}
$$

If $\nabla$ has no parameters we drop the prefix ' $p$-'. A logic L is called ( $\mathrm{p}-$ )disjunctional if there is a (p-)disjunction in $\mathrm{L}$.

We know from [13, Theorem 2.5.17] that every finitary protoalgebraic distributive logic is pdisjunctional. ${ }^{9}$ Therefore, from this result we could already obtain that SL (and its axiomatic extensions) is p-disjunctional. Indeed algebraizable logics form a subclass of protoalgebraic logics, and all logics considered in the paper are distributive (see the preliminaries). However, here we can do better by providing an explicit, reasonably simple, description of the p-disjunction, which then can be used to obtain many consequences by applying general AAL theorems. Another advantage of our approach is that it is applicable to all substructural logics, not just to axiomatic extensions of SL.

Our approach is based on [12, Theorem 2.6.9] which shows that certain sets of basic deductive terms in an almost (MP)-based substructural logic determine a p-disjunction of a given logic. Here we prove a stronger version of that theorem by removing those conditions at the price of a (seemingly) slightly more complicated form of the resulting p-disjunction. By 'seemingly' we mean that in the majority of substructural logics we study in this paper this complication is actually nonexistent.

Theorem 21. Let $\mathrm{L}$ be an almost (MP)-based substructural logic with a set of basic deduction terms bDT. Then the set $\nabla_{\mathrm{L}}=\left\{\gamma_{1}(p) \vee \gamma_{2}(q) \mid \gamma_{1}, \gamma_{2} \in(\mathrm{bDT} \cup\{\star \wedge \overline{1}\})^{*}\right\}$ is a p-disjunction in $\mathrm{L}$.

Proof. Clearly the set bDT $\cup\{\star \wedge \overline{1}\}$ is a set of basic deduction terms (because already the logic SL proves $\left(\mathrm{Adj}_{\mathrm{u}}\right)$ and $\left.\left(\mathrm{P}_{\mathrm{SL}} 24\right)\right)$. Therefore $\nabla_{\mathrm{L}}$ obviously satisfy the condition $(\mathrm{PD})$; we prove that it satisfies PCP as well.

Assume that $\Gamma, \varphi \vdash_{\mathrm{L}} \chi$ and $\Gamma, \psi \vdash_{\mathrm{L}} \chi$. From Corollary 18 we obtain $\delta_{\varphi}, \delta_{\psi} \in \Pi\left((\mathrm{bDT} \cup\{\star \wedge \overline{1}\})^{*}\right)$ such that $\Gamma \vdash_{\mathrm{L}} \delta_{\varphi}(\varphi) \rightarrow \chi$ and $\Gamma \vdash_{\mathrm{L}} \delta_{\psi}(\psi) \rightarrow \chi$. Thus also $\Gamma \vdash_{\mathrm{L}} \delta_{\varphi}(\varphi) \wedge \overline{1} \rightarrow \chi$ and $\Gamma \vdash_{\mathrm{L}}$ $\delta_{\psi}(\psi) \wedge \overline{1} \rightarrow \chi($ due to $(\wedge 1)$ and $(\mathrm{T}))$ and so, without a loss of generality, we might assume that the outmost term in $\delta_{\varphi}$ and $\delta_{\psi}$ is $\star \wedge \overline{1}$ and so we have $\vdash_{\mathrm{L}} \delta(\varphi) \& \psi \rightarrow \psi$ and $\vdash_{\mathrm{L}} \psi \& \delta(\varphi) \rightarrow \psi$ (due to $\left(\mathrm{P}_{\mathrm{SL}} 22\right)$ and $\left(\mathrm{P}_{\mathrm{SL}} 23\right)$ ) for both $\delta=\delta_{\varphi}$ and $\delta=\delta_{\psi}$.

We also know that $\Gamma \vdash_{\mathrm{L}} \delta_{\varphi}(\varphi) \vee \delta_{\psi}(\psi) \rightarrow \chi$ by $(\vee 3)$. The proof is done by showing by induction over the sum of the depths of the trees representing $\delta_{\varphi}, \delta_{\psi}$ that:

$$
\varphi \nabla_{\mathrm{L}} \psi \vdash_{\mathrm{L}} \delta_{\varphi}(\varphi) \vee \delta_{\psi}(\psi)
$$

The base of induction (when $\delta_{\varphi}, \delta_{\psi} \in \mathrm{bDT} \cup\{\star \wedge \overline{1}\}$ ) is trivial. For the induction step assume that $\delta_{\psi}=\delta_{1} \& \delta_{2}$. Using $\left(\mathrm{P}_{\mathrm{SL}} 20\right),\left(\mathrm{P}_{\mathrm{SL}} 21\right),(\vee 1),(\vee 2)$, and $(\vee 3)$ we obtain the following chain of implications:

\footnotetext{
${ }^{9}$ In [10, Theorem 4.25] the result it is generalized to all protoalgebraic distributive logics.
} 


\begin{tabular}{|l|l|l|}
\hline Logic $\mathrm{L}$ & $\mathrm{bDT}_{\mathrm{L}}$ & $(\mathrm{p}-)$ disjunction $\nabla_{\mathrm{L}}$ in L \\
\hline \hline $\mathrm{SL}$ & $\left\{\alpha_{\delta, \varepsilon}, \alpha_{\delta, \varepsilon}^{\prime}, \beta_{\delta, \varepsilon}, \beta_{\delta, \varepsilon}^{\prime}, \star \wedge \overline{1}\right\} \mid \delta, \varepsilon$ formulae $\}$ & $\left\{\gamma_{1}(p) \vee \gamma_{2}(q) \mid \gamma_{1}, \gamma_{2} \in \mathrm{bDT}_{\mathrm{SL}}\right\}$ \\
\hline $\mathrm{SL}_{\mathrm{a}}$ & $\left\{\lambda_{\varepsilon}, \rho_{\varepsilon}, \star \wedge \overline{1} \mid \varepsilon\right.$ a formula $\}$ & $\left\{\gamma_{1}(p) \vee \gamma_{2}(q) \mid \gamma_{1}, \gamma_{2} \in \mathrm{bDT}_{\mathrm{SL}_{\mathrm{a}}}\right\}$ \\
\hline $\mathrm{SL}_{\mathrm{ae}}$ & $\{\star \wedge \overline{1}\}$ & $\{(\varphi \wedge \overline{1}) \vee(\psi \wedge \overline{1})\}$ \\
\hline $\mathrm{SL}_{\mathrm{aew}}$ & $\{\star\}$ & $\{\varphi \vee \psi\}$ \\
\hline
\end{tabular}

Table 5: (p-)disjunctions in prominent substructural logics

$$
\begin{gathered}
\left(\delta_{\varphi}(\varphi) \vee \delta_{1}(\psi)\right) \&\left(\delta_{\varphi}(\varphi) \vee \delta_{2}(\psi)\right) \rightarrow \\
\rightarrow\left[\delta_{\varphi}(\varphi) \& \delta_{\varphi}(\varphi)\right] \vee\left[\delta_{\varphi}(\varphi) \& \delta_{2}(\psi)\right] \vee\left[\delta_{1}(\psi) \& \delta_{\varphi}(\varphi)\right] \vee\left[\delta_{1}(\psi) \& \delta_{2}(\psi)\right] \rightarrow \\
\rightarrow \delta_{\varphi}(\varphi) \vee \delta_{\varphi}(\varphi) \vee \delta_{\varphi}(\varphi) \vee\left[\delta_{1}(\psi) \& \delta_{2}(\psi)\right] \rightarrow \delta_{\varphi}(\varphi) \vee \delta_{\psi}(\psi) .
\end{gathered}
$$

The induction assumption used for $\delta_{\varphi}(\varphi) \vee \delta_{1}(\psi)$ and $\delta_{\varphi}(\varphi) \vee \delta_{2}(\psi)$ together with $(\operatorname{Adj} \&)$ completes the proof.

If $\mathrm{bDT}^{*}$ contains a formula $\delta$ such that $\vdash_{\mathrm{L}} \delta \leftrightarrow \star \wedge \overline{1}$ (which is the case in all the logics we study in this paper) we can omit the extra formula $\star \wedge \overline{1}$ from the formulation of the above theorem. Therefore we can simplify the description of p-disjunctions in these logics; see Table 5 (also note that for each $\gamma \in \mathrm{bDT}_{\mathrm{SL}_{\mathrm{ae}}}$ we have, using $\left.\left(\mathrm{P}_{\mathrm{SL}} 28\right), \vdash_{\mathrm{SL}_{\mathrm{ae}}} \gamma \leftrightarrow \star \wedge \overline{1}\right)$.

Let us now present the promised applications of having a p-disjunction in a logic. We start with the description of intersections of filters.

Theorem 22 ([10, Theorem 4.7]). For each SL-algebra $\boldsymbol{A}$ and each $X, Y \subseteq A$ we have $\operatorname{Fi}(X) \cap$ $\operatorname{Fi}(Y)=\operatorname{Fi}\left(X \nabla_{\mathrm{SL}}^{\boldsymbol{A}} Y\right)$.

Of course, if $\boldsymbol{A}$ is in a subquasivariety of some substructural logic with a simpler p-disjunction $\nabla$, this result can be accordingly simplified.

The second application concerns the axiomatization of substructural logics given by special classes of SL-algebras. Recall that in first-order logic a positive clause $C$ is a disjunction of finitely-many atomic formulae. We define a positive equational clause as a disjunction of finitelymany equations $C=\bigvee_{i \in \mathcal{I}_{C}} \delta_{i} \approx \varepsilon_{i}$. A set of positive equational clauses $\mathcal{C}$ is said to be valid in an SL-algebra $\boldsymbol{A}$, written as $\boldsymbol{A}=\mathcal{C}$, if for each $C \in \mathcal{C}$ and each $\boldsymbol{A}$-evaluation $e$ there is $i \in \mathcal{I}_{C}$ such that $e\left(\delta_{i}\right)=e\left(\varepsilon_{i}\right)$; a set of algebras satisfying certain set of positive equational clauses is called a positive universal class. Theorem 23 shows how to axiomatize substructural logics given by positive equational classes of SL-algebras. This theorem and its consequences generalize the results in [16] for associative substructural logics (there formulated for varieties of FL-algebras).

Theorem 23. Let $\mathrm{L}$ be a substructural logic with a p-disjunction $\nabla$, and let $\mathcal{C}$ be a set of positive equational clauses. Then:

$$
\models_{\{\boldsymbol{A} \in \mathbb{L} \mid \boldsymbol{A} \models \mathcal{C}\}}=\mathrm{L}+\bigcup\left\{\nabla_{i \in \mathcal{I}_{C}}\left(\delta_{i} \leftrightarrow \varepsilon_{i}\right) \mid C \in \mathcal{C}\right\} .
$$

Proof. Direct application of [10, Theorem 5.7].

Note that if the set of positive equational clauses is recursive, so it is the axiomatization of its corresponding logic. As a corollary we obtain a way to axiomatize intersections of axiomatic extensions of a given logic (again, see [10] for the detailed general formulation).

Corollary 24. Let $\mathrm{L}$ be a substructural logic with a p-disjunction $\nabla$, and let $\mathrm{L}_{1}, \mathrm{~L}_{2}$ be axiomatic extensions of $\mathrm{L}$ by sets of axioms $A X_{1}$ and $A X_{2}$, respectively. Without loss of generality we can assume that $A X_{1}$ and $A X_{2}$ are written in disjoint sets of variables. Then:

$$
\mathrm{L}_{1} \cap \mathrm{L}_{2}=\mathrm{L}+\bigcup\left\{\varphi \nabla \psi \mid \varphi \in A X_{1} \text { and } \psi \in A X_{2}\right\} .
$$


Equivalently, the theorem and its corollary can be dualized as a description of the variety of SLalgebras generated by a positive universal class and as an effective method to compute equational bases for joins of relative subvarieties of a given quasivariety of SL-algebras.

Corollary 25. Let $\mathcal{C}$ be a set of positive equational clauses. Then an equational base for the variety of SL-algebras generated by those satisfying $\mathcal{C}$ can be obtained by adding the following:

$$
\overline{1} \approx \overline{1} \wedge\left[\nabla_{i \in \mathcal{I}_{C}}\left(\delta_{i} \leftrightarrow \varepsilon_{i}\right)\right] \text { for each } C \in \mathcal{C} .
$$

Corollary 26. Let $\mathbb{L}$ be a quasivariety of SL-algebras, $\nabla$ a p-disjunction for the corresponding logic, and let $\mathbb{L}_{1}, \mathbb{L}_{2}$ be relative subvarieties of $\mathbb{L}$ given by sets of equations $\mathcal{E}_{1}$ and $\mathcal{E}_{2}$, respectively. Without loss of generality we can assume that $\mathcal{E}_{1}$ and $\mathcal{E}_{2}$ are written in disjoint sets of variables. Then:

$$
\mathbb{L}_{1} \vee \mathbb{L}_{2}=\mathbb{L}+\bigcup\left\{\left(\left(\delta_{1} \leftrightarrow \varepsilon_{1}\right) \nabla\left(\delta_{2} \leftrightarrow \varepsilon_{2}\right)\right) \wedge \overline{1} \approx \overline{1} \mid \delta_{1} \approx \varepsilon_{1} \in \mathcal{E}_{1} \text { and } \delta_{2} \approx \varepsilon_{2} \in \mathcal{E}_{2}\right\} .
$$

Observe that this result can be generalized to joins of finitely-many relative subvarieties (as well as the previous one extends to intersection of finitely-many axiomatic extensions). In particular, we obtain that the join of finitely-many recursively based relative subvarieties is recursively based.

\section{Semilinear substructural logics}

This section is devoted to semilinear extensions of substructural logics. The notion of semilinear logic has been introduced in the very general setting of weakly p-implicational logics in [11] and systematically used as a general framework for study of mathematical fuzzy logics in [12]. Let us first recall four equivalent (in the present context) definitions of semilinear logic: the first one is the original definition, the second is a purely syntactical characterization called Semilinear Property SLP, the third one is also syntactical and it is based on the well-known prelinearity axiom and the behavior of lattice disjunction as a proper disjunction, and the last one is purely semantical. The last one also stands behind the name 'semilinear' as explained in the introduction.

Definition 27. Let $\mathrm{L}$ be a substructural logic and $\mathbb{K}$ the class of all L-chains. We say that $\mathrm{L}$ is semilinear if one of the following equivalent conditions is met:

- For every set of formulae $\Gamma$ and every formula $\varphi$ we have:

$$
\Gamma \vdash_{\mathrm{L}} \varphi \quad \text { if, and only if, } \quad \Gamma \models_{\mathbb{K}} \varphi \text {. }
$$

- For every set of formulae $\Gamma$ and every formulae $\varphi, \psi, \chi$ we have:

$$
\Gamma, \varphi \rightarrow \psi \vdash_{\mathrm{L}} \chi \quad \text { and } \quad \Gamma, \psi \rightarrow \varphi \vdash_{\mathrm{L}} \chi \quad \text { imply } \quad \Gamma \vdash_{\mathrm{L}} \chi .
$$

- $\vee$ satisfies prelinearity and the Proof by Cases Property, i.e. for every set of formulae $\Gamma$ and every formulae $\varphi, \psi, \chi$ we have:

$$
\begin{gathered}
\vdash_{\mathrm{L}}(\varphi \rightarrow \psi) \vee(\psi \rightarrow \varphi) \\
\Gamma, \varphi \vdash_{\mathrm{L}} \chi \quad \text { and } \quad \Gamma, \psi \vdash_{\mathrm{L}} \chi \quad \text { imply } \quad \Gamma, \varphi \vee \psi \vdash_{\mathrm{L}} \chi
\end{gathered}
$$

- $\mathbb{K}$ is the class of all relatively finitely subdirectly irreducible L-algebras.

In the first subsection we show several equivalent ways to axiomatize the minimum semilinear logic extending a given almost (MP)-based substructural logic. Generalizing the work done in [12] we make a heavy use of the p-disjunction to produce the axiomatization, namely we write it in terms of the corresponding set bDT. In the second subsection we prove that these semilinear extensions are also complete with respect to distinguished classes of chains, namely those over real and rational unit interval. 


\subsection{Axiomatization of semilinear extensions}

Although, the logic $\mathrm{L}^{\ell}$ is primarily defined in [11] as the weakest semilinear logic extending L, the next definition formalizes this notion in the form suitable for this paper by using an 'implicit' Hilbert-style axiomatic system.

Definition 28. Let $\mathrm{L}$ be a substructural logic and $\mathbb{K}$ the class of $\mathrm{L}$-chains. We define the logic $\mathrm{L}^{\ell}$ as the extension of $\mathrm{L}$ by all the rules $\Gamma \vdash_{\mathrm{L}^{\ell}} \varphi$ for which holds $\Gamma \models_{\mathbb{K}} \varphi$.

The general theory explained in $[12]$ gives us two immediate ways how to axiomatize $\mathrm{L}^{\ell}$ in some better/simpler way (assuming that L is almost (MP)-based). They appear in the next theorem as alternatives $\mathrm{A}$ and $\mathrm{B}$. Both these alternatives have some advantages but are unnecessary complicated: the first one adds only axioms but needs to use all iterated deductive terms, whereas the other one uses only basic terms but adds new rules. We show that in the case of substructural logics we can obtain a third and a fourth alternative combining the advantages of the first two (we present these two variants because they generalize two different usual formulations appearing in the literature).

Theorem 29. Let $\mathrm{L}$ be an almost (MP)-based substructural logic with a set $\mathrm{bDT}$ of basic deductive terms. Then $\mathrm{L}^{\ell}$ is axiomatized, relatively to $\mathrm{L}$, by any of the following four sets of axioms/rules:

$$
\begin{array}{ll}
A & \gamma_{1}(\varphi \rightarrow \psi) \vee \gamma_{2}(\psi \rightarrow \varphi), \text { for every } \gamma_{1}, \gamma_{2} \in(\mathrm{bDT} \cup\{\star \wedge \overline{1}\})^{*} \\
B & (\varphi \rightarrow \psi) \vee(\psi \rightarrow \varphi) \\
& (\varphi \rightarrow \psi) \vee \chi, \varphi \vee \chi \vdash \psi \vee \chi \\
& \varphi \vee \psi \vdash \gamma(\varphi) \vee \psi, \text { for every } \gamma \in \mathrm{bDT} \\
C & ((\varphi \rightarrow \psi) \wedge \overline{1}) \vee \gamma((\psi \rightarrow \varphi) \wedge \overline{1}), \text { for every } \gamma \in \mathrm{bDT} \cup\{\star\} \\
D & (\varphi \vee \psi \rightarrow \psi) \vee \gamma(\varphi \vee \psi \rightarrow \psi), \text { for every } \gamma \in \mathrm{bDT} \cup\{\star \wedge \overline{1}\} .
\end{array}
$$

Proof. For each $X \in\{A, B, C, D\}$ we denote as $\mathrm{L}_{X}$ the corresponding extension of L. Using Theorem 21 we know that $\left\{\gamma_{1}(p) \vee \gamma_{2}(q) \mid \gamma_{1}, \gamma_{2} \in(\mathrm{bDT} \cup\{\star \wedge \overline{1}\})^{*}\right\}$ is a p-disjunction in L. Therefore $\mathrm{L}_{A}=\mathrm{L}^{\ell}$ due to [12, Theorem 3.2.1]. To show that $\mathrm{L}_{B}=\mathrm{L}^{\ell}$ just use [12, Proposition 3.2.9] and [12, Theorem 2.7.27].

To complete the proof we will show the following chain of inclusions: $\mathrm{L}^{\ell} \supseteq \mathrm{L}_{C} \supseteq \mathrm{L}_{D} \supseteq \mathrm{L}_{B}$. For the first one take $\gamma \in \mathrm{bDT} \cup\{\star\}$; then we have:

(a) $\varphi \rightarrow \psi \vdash_{\mathrm{L}^{\ell}}((\varphi \rightarrow \psi) \wedge \overline{1}) \vee \gamma((\psi \rightarrow \varphi) \wedge \overline{1})$

(b) $\psi \rightarrow \varphi \vdash_{\mathrm{L}^{\ell}} \gamma((\psi \rightarrow \varphi) \wedge \overline{1})$

(c) $\psi \rightarrow \varphi \vdash_{\mathrm{L}^{\ell}}((\varphi \rightarrow \psi) \wedge \overline{1}) \vee \gamma((\psi \rightarrow \varphi) \wedge \overline{1})$

(d) $\vdash_{\mathrm{L}^{\ell}}((\varphi \rightarrow \psi) \wedge \overline{1}) \vee \gamma((\psi \rightarrow \varphi) \wedge \overline{1})$

$\left(\operatorname{Adj}_{\mathrm{u}}\right),(\vee 1)$, and $(\mathrm{MP})$

$\left(\operatorname{Adj}_{\mathrm{u}}\right)$ and $\varphi \vdash \gamma(\varphi)$

(b), ( $\vee 2)$, and (MP)

Next we prove the second inclusion, let us first assume that $\gamma \in$ bDT:

(a) $\vdash_{\mathrm{L}_{C}}(\varphi \rightarrow \psi) \wedge \overline{1} \rightarrow(\varphi \vee \psi \rightarrow \psi) \vee \gamma(\varphi \vee \psi \rightarrow \varphi)$

(a), (c), and $\operatorname{SLP}^{10}$

(b) $\vdash_{\mathrm{L}_{C}} \gamma^{\prime}((\psi \rightarrow \varphi) \wedge \overline{1}) \rightarrow \gamma(\varphi \vee \psi \rightarrow \varphi)$

$\left(\mathrm{P}_{\mathrm{SL}} 26\right),(\mathrm{V} 1)$, and $(\mathrm{T})$

(c) $\vdash_{\mathrm{L}_{C}} \gamma^{\prime}((\psi \rightarrow \varphi) \wedge \overline{1}) \rightarrow(\varphi \vee \psi \rightarrow \psi) \vee \gamma(\varphi \vee \psi \rightarrow \varphi)$

$\left(\mathrm{P}_{\mathrm{SL}} 27\right)$ and Lemma 16

(d) $\vdash_{\mathrm{L}_{C}}((\varphi \rightarrow \psi) \wedge \overline{1}) \vee \gamma^{\prime}((\psi \rightarrow \varphi) \wedge \overline{1}) \rightarrow(\varphi \vee \psi \rightarrow \psi) \vee \gamma(\varphi \vee \psi \rightarrow \varphi)$

(b), ( $\vee 2)$, and $(\mathrm{T})$

(e) $\vdash_{\mathrm{L}_{C}}(\varphi \vee \psi \rightarrow \psi) \vee \gamma(\varphi \vee \psi \rightarrow \varphi)$

(a), (c), and ( $\mathrm{V} 3)$

(d) and (MP)

The proof for $\gamma=\star \wedge \overline{1}$ is analogous: in step (b) we would set $\gamma^{\prime}=\star$ and prove it using $\left(\mathrm{P}_{\mathrm{SL}} 27\right)$, $\left(\mathrm{Adj}_{\mathrm{u}}\right),\left(\mathrm{P}_{\mathrm{SL}} 24\right),(\mathrm{MP}),\left(\mathrm{P}_{\mathrm{SL}} 28\right)$, and $(\mathrm{T})$. To prove the last inclusion we first show that $\mathrm{L}_{D}$ proves prelinearity:

\footnotetext{
${ }^{10}$ Clearly, as $\mathrm{L}^{\ell}$ is a semilinear logic we know it satisfies the Semilinear Property, see Definition 27.
} 
(a) $\vdash_{\mathrm{L}_{D}}(\varphi \vee \psi \rightarrow \psi) \rightarrow(\varphi \rightarrow \psi)$

$(\mathrm{V} 1)$ and $(\mathrm{Sf})$

(b) $\vdash_{\mathrm{L}_{D}}(\varphi \vee \psi \rightarrow \psi) \rightarrow(\varphi \rightarrow \psi) \vee(\psi \rightarrow \varphi)$

(a), ( 1$)$, and (T)

c) $\vdash_{\mathrm{L}_{D}}(\varphi \vee \psi \rightarrow \varphi) \rightarrow(\varphi \rightarrow \psi) \vee(\psi \rightarrow \varphi)$

analogously

(d) $\vdash_{\mathrm{L}_{D}}(\varphi \vee \psi \rightarrow \varphi) \wedge \overline{1} \rightarrow(\varphi \rightarrow \psi) \vee(\psi \rightarrow \varphi)$

$(\mathrm{c}),(\wedge 1)$, and $(\mathrm{T})$

(e) $\vdash_{\mathrm{L}_{D}}(\varphi \vee \psi \rightarrow \psi) \vee((\varphi \vee \psi \rightarrow \varphi) \wedge \overline{1}) \rightarrow(\varphi \rightarrow \psi) \vee(\psi \rightarrow \varphi)$

(b), (d), and ( $\mathrm{V} 3)$

(f) $\vdash_{\mathrm{L}_{D}}(\varphi \rightarrow \psi) \vee(\psi \rightarrow \varphi)$

(e) and (MP)

Next we show $\varphi \vee \psi \vdash_{\mathrm{L}_{D}} \gamma(\varphi) \vee \psi$ for each $\gamma \in$ bDT:

(a) $\varphi \vee \psi \vdash_{\mathrm{L}_{D}}(\varphi \vee \psi \rightarrow \psi) \rightarrow \psi$

(b) $\varphi \vee \psi \vdash_{\mathrm{L}_{D}}(\varphi \vee \psi \rightarrow \psi) \rightarrow \gamma(\varphi) \vee \psi$

(a), $(\mathrm{V} 2)$, and $(\mathrm{T})$

(c) $\varphi \vee \psi \vdash_{\mathrm{L}_{D}}(\varphi \vee \psi \rightarrow \varphi) \rightarrow \varphi$

(d) $\varphi \vee \psi \vdash_{\mathrm{L}_{D}} \gamma^{\prime}(\varphi \vee \psi \rightarrow \varphi) \rightarrow \gamma(\varphi)$

(c) and Lemma 16

(e) $\varphi \vee \psi \vdash_{\mathrm{L}_{D}} \gamma^{\prime}(\varphi \vee \psi \rightarrow \varphi) \rightarrow \gamma(\varphi) \vee \psi$

(d), (V1), and (T)

(f) $\varphi \vee \psi \vdash_{\mathrm{L}_{D}}(\varphi \vee \psi \rightarrow \psi) \vee \gamma^{\prime}(\varphi \vee \psi \rightarrow \varphi) \rightarrow \gamma(\varphi) \vee \psi$

(b), (e), and ( $\mathrm{V} 3)$

(g) $\varphi \vee \psi \vdash_{\mathrm{L}_{D}} \gamma(\varphi) \vee \psi$

(f) and (MP)

Note that the same proof would work for $\gamma=\star \wedge \overline{1}$; only in step (d) we would set $\gamma^{\prime}=\star \wedge \overline{1}$ and prove it from (c) using $\left(\operatorname{Adj}_{\mathrm{u}}\right),\left(\mathrm{P}_{\mathrm{SL}} 24\right)$, and (MP). Thus we know that $\varphi \vee \psi \vdash_{\mathrm{L}_{D}}(\varphi \wedge \overline{1}) \vee \psi$ which we use to prove now $(\varphi \rightarrow \psi) \vee \chi, \varphi \vee \chi \vdash \psi \vee \chi$ :

(a) $\varphi \vee \chi \vdash \chi \rightarrow \psi \vee \chi$

(b) $\vdash_{\mathrm{L}_{D}}(\varphi \rightarrow \psi) \wedge \overline{1} \rightarrow(\varphi \vee \chi \rightarrow \psi \vee \chi)$

(c) $\vdash_{\mathrm{L}_{D}} \varphi \vee \chi \rightarrow((\varphi \rightarrow \psi) \wedge \overline{1} \rightsquigarrow \psi \vee \chi)$

(d) $\varphi \vee \chi \vdash_{\mathrm{L}_{D}}(\varphi \rightarrow \psi) \wedge \overline{1} \rightarrow \psi \vee \chi$

(c), (MP), and $\left(\mathrm{Symm}_{1}\right)$

(e) $\varphi \vee \chi \vdash_{\mathrm{L}_{D}}((\varphi \rightarrow \psi) \wedge \overline{1}) \vee \chi \rightarrow \psi \vee \chi$

(a), (d), and ( $\mathrm{V} 3)$

(f) $\quad(\varphi \rightarrow \psi) \vee \chi \vdash_{\mathrm{L}_{D}}((\varphi \rightarrow \psi) \wedge \overline{1}) \vee \chi$ see the previous paragraph

(g) $(\varphi \rightarrow \psi) \vee \chi, \varphi \vee \chi \vdash_{\mathrm{L}_{D}} \psi \vee \chi$

(e), (f), and (MP)

Table 6 collects axiomatizations of important semilinear substructural logics obtained as axiomatization $\mathrm{C}$ from Theorem 29. We present them in the form of axiom schemata, sometimes altered a little for simplicity or to obtain some form known from the literature. These simplifications follow from the following few simple observations:

- In the logics with weakening we use the fact that $\vdash_{\mathrm{SL}_{\mathrm{w}}} \varphi \leftrightarrow \varphi \wedge \overline{1}$ to work with the axiomatization $C^{\prime}(\varphi \rightarrow \psi) \vee \gamma(\psi \rightarrow \varphi)$, for every $\gamma \in \operatorname{bDT} \cup\{\star\}$.

- The axiom for $\gamma=\star \wedge \overline{1}$ can be omitted from all axiomatizations because it follows from the one for $\gamma=\star$ using $\left(\mathrm{P}_{\mathrm{SL}} 28\right)$.

- The axiom for $\gamma=\star$ can be omitted from all but the last two axiomatizations because it follows from the one for $\alpha_{\overline{1}, \overline{1}}$ (or $\lambda_{\overline{1}}$ ) using the first (or also the fifth) claim of Proposition 10.

- In the case of $\mathrm{SL}_{\mathrm{e}}$, we first note that the proposed single formula to axiomatize $\mathrm{SL}_{\mathrm{e}}^{\ell}$ is an instance of formulae from axiomatization $\mathrm{A}$. On the other hand, setting $\delta=\varepsilon=\overline{1}$ or respectively $\delta^{\prime}=\varepsilon^{\prime}=\overline{1}$ and using the first claim of Proposition 10, we obtain the remaining two axioms from axiomatization $\mathrm{C}$.

- In $\mathrm{SL}_{\mathrm{a}}$ we proceed analogously to the previous case. 


\begin{tabular}{|l|l|}
\hline Logic L & additional axioms needed to axiomatize $\mathrm{L}^{\ell}$ \\
\hline \hline $\mathrm{SL}$ & $((\varphi \rightarrow \psi) \wedge \overline{1}) \vee \gamma((\psi \rightarrow \varphi) \wedge \overline{1})$, for every $\gamma \in\left\{\alpha_{\delta, \varepsilon}, \alpha_{\delta, \varepsilon}^{\prime}, \beta_{\delta, \varepsilon}, \beta_{\delta, \varepsilon}^{\prime}\right\}$ \\
\hline $\mathrm{SL}_{\mathrm{w}}$ & $(\varphi \rightarrow \psi) \vee \gamma(\psi \rightarrow \varphi)$, for every $\gamma \in\left\{\alpha_{\delta, \varepsilon}, \alpha_{\delta, \varepsilon}^{\prime}, \beta_{\delta, \varepsilon}, \beta_{\delta, \varepsilon}^{\prime}\right\}$ \\
\hline $\mathrm{SL}_{\mathrm{e}}$ & $\alpha_{\delta, \varepsilon}((\varphi \rightarrow \psi) \wedge \overline{1}) \vee \beta_{\delta^{\prime}, \varepsilon^{\prime}}((\psi \rightarrow) \wedge \overline{1})$ \\
\hline $\mathrm{SL}_{\mathrm{ew}}$ & $\alpha_{\delta, \varepsilon}(\varphi \rightarrow \psi) \vee \beta_{\delta^{\prime}, \varepsilon^{\prime}}(\psi \rightarrow \varphi)$ \\
\hline $\mathrm{SL}_{\mathrm{a}}$ & $\left(\lambda_{\varepsilon}(\varphi \rightarrow \psi) \wedge \overline{1}\right) \vee\left(\rho_{\varepsilon^{\prime}}(\psi \rightarrow \varphi) \wedge \overline{1}\right)$ \\
\hline $\mathrm{SL}_{\mathrm{ae}}$ & $((\varphi \rightarrow \psi) \wedge \overline{1}) \vee((\psi \rightarrow \varphi) \wedge \overline{1})$ \\
\hline $\mathrm{SL}_{\mathrm{aew}}$ & $(\varphi \rightarrow \psi) \vee(\psi \rightarrow \varphi)$ \\
\hline
\end{tabular}

Table 6: Axiomatization of $\mathrm{L}^{\ell}$ for prominent substructural logics

\subsection{Completeness properties}

Next we prove that the non-associative semilinear logics axiomatized above are not only complete with respect to the semantics of all their chains, but also with respect to some distinguished classes of chains, namely those defined over the rational and real unit interval (standard completeness). In fact, we will prove completeness in the following strong sense.

Definition 30. Let $\mathrm{L}$ be a substructural semilinear logic and $\mathbb{K}$ a class of L-chains. We say that $\mathrm{L}$ has the property of strong $\mathbb{K}$-completeness, $\mathrm{SKC}$ for short, when for every set of formulae $\Gamma \cup\{\varphi\}, \Gamma \vdash_{\mathrm{L}} \varphi$ if, and only if, $\Gamma \models_{\mathbb{K}} \varphi$.

We will need the following characterization of $\mathrm{SKC}$ (given in general in [12, Theorem 3.4.6]).

Theorem 31. Let $\mathrm{L}$ be a substructural semilinear logic and $\mathbb{K}$ a class of $\mathrm{L}$-chains. Then $\mathrm{L}$ has the $\mathrm{SKC}$ if, and only if, every countable nontrivial L-chain is embeddable into a member of $\mathbb{K}$.

Let $\mathrm{S} \subseteq\{\mathrm{e}, \mathrm{c}, \mathrm{i}, \mathrm{o}\}$. In the light of Definition 30 we define the class $\mathcal{Q}$ (resp. $\mathcal{R}$ ) of all $\mathrm{SL}_{\mathrm{S}}$-chains whose universe is the rational unit interval $Q \cap[0,1]$ (resp. the real unit interval $[0,1]$ ). Note that if $\boldsymbol{A}$ is in $\mathcal{Q}$ or $\mathcal{R}$ then $0^{\boldsymbol{A}}, 1^{\boldsymbol{A}}$ need not coincide with the real numbers 0,1 which play the role of $\perp$ and $T$. They coincide iff $\{\mathrm{i}, \mathrm{o}\} \subseteq \mathrm{S}$. The remaining part of the paper is devoted to the proof of the following theorem.

Theorem 32. Let $\mathrm{S} \subseteq\{\mathrm{e}, \mathrm{c}, \mathrm{i}, \mathrm{o}\}$. Then the logic $\mathrm{SL}_{\mathrm{S}}^{\ell}$ has the $\mathrm{S} \mathcal{Q C}$ and $\mathrm{SRC}$.

Before we prove Theorem 32, we introduce several auxiliary constructions which we will need in its proof. Let $\langle A, \leq\rangle$ be a chain and $a, b \in A$. We denote the fact that $a$ is a subcover of $b$ as $a \prec b$, i.e., $a \prec b$ holds iff $a<b$ and there is no $c \in A$ such that $a<c<b$. A chain $\langle A, \leq\rangle$ is said to be dense if $a \prec b$ does not hold for any $a, b \in A$.

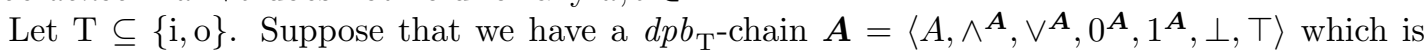
countable and nontrivial (i.e. has at least two elements). We show that it is possible to extend $\boldsymbol{A}$ to a dense $d p b_{\mathrm{T}}$-chain $\boldsymbol{D}$. If $\boldsymbol{A}$ is not dense then there is at least one element $a$ which has a subcover $a^{\prime}$. As we want to extend $\boldsymbol{A}$ so that it becomes dense, we have to fill for each such element $a$ the gap between $a$ and $a^{\prime}$ by a countable dense chain. This can be done by pasting a copy of rational numbers (namely $\mathrm{Q} \cap(0,1))$ into the gap between $a$ and $a^{\prime}$. (see Figure 1) Formally we can define the set $D$ as the following subset of $A \times(Q \cap(0,1])$ :

$$
D=\{\langle a, 1\rangle \mid a \in A\} \cup\left\{\langle a, q\rangle \mid q \in \mathrm{Q} \cap(0,1) \text { and }\left(\exists a^{\prime} \in A\right) \text { such that } a^{\prime} \prec a\right\} .
$$

Then the lexicographic order $\leq_{\operatorname{lex}}$ on $D$ is a dense linear order, $\langle\top, 1\rangle$ is a top element, and $\langle\perp, 1\rangle$ is a bottom element. Thus the algebra $\boldsymbol{D}=\left\langle D, \wedge^{\boldsymbol{D}}, \vee^{\boldsymbol{D}},\left\langle 0^{\boldsymbol{A}}, 1\right\rangle,\left\langle 1^{\boldsymbol{A}}, 1\right\rangle,\langle\perp, 1\rangle,\langle\top, 1\rangle\right\rangle$, where $\wedge^{\boldsymbol{D}}$ and $\vee^{\boldsymbol{D}}$ are defined by $\leq_{\text {lex }}$, is a $d p b$-chain. Moreover, if $1^{\boldsymbol{A}}=\top$ then $\left\langle 1^{\boldsymbol{A}}, 1\right\rangle=\langle\top, 1\rangle$. Similarly, $\left\langle 0^{\boldsymbol{A}}, 1\right\rangle=\langle\perp, 1\rangle$ if $0^{\boldsymbol{A}}=\perp$. Hence $\boldsymbol{D}$ is even a $d p b_{\mathrm{T}^{-}}$chain. Finally, it is clear that the subset $A \times\{1\} \subseteq D$ forms a $d p b_{\mathrm{T}^{-}}$-chain isomorphic to $\boldsymbol{A}$. 


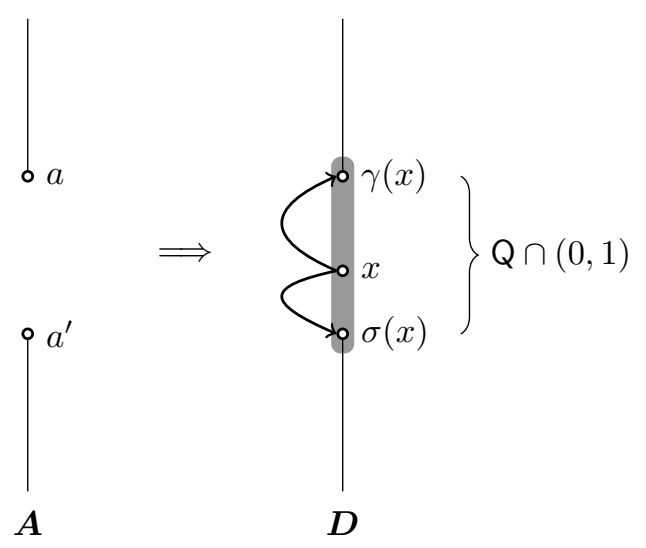

Figure 1: Filling gaps.

Observe that we can define two operators on the chain $\boldsymbol{D}$ whose image is $A \times\{1\}$, namely a closure operator $\gamma$ and an interior operator $\sigma$ defined as follows:

$$
\begin{aligned}
\gamma(a, q) & =\langle a, 1\rangle, \\
\sigma(a, q) & = \begin{cases}\langle a, 1\rangle & \text { if } q=1, \\
\left\langle a^{\prime}, 1\right\rangle & \text { if } q<1 \text { and } a^{\prime} \prec a .\end{cases}
\end{aligned}
$$

Note that $A \times\{1\}$ is the set of $\gamma$-closed and $\sigma$-open elements. Summing up, if we identify $A$ with $A \times\{1\}$, we obtain the following general lemma.

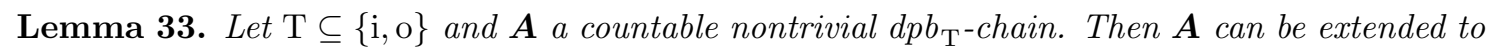
a countably infinite dense $d p b_{\mathrm{T}}$-chain $\boldsymbol{D}$. Moreover, there are a closure operator $\gamma$ and an interior operator $\sigma$ on $\boldsymbol{D}$ such that $A=\gamma[D]=\sigma[D]$.

Next we introduce a construction allowing us to combine two $r t_{\mathrm{S}}$-groupoids on a same chain together. Let $\mathrm{S} \subseteq\{\mathrm{e}, \mathrm{c}, \mathrm{i}, \mathrm{o}\}$ and $\mathrm{T}=\mathrm{S} \backslash\{\mathrm{e}, \mathrm{c}\}$. Assume that we have two different $r \ell_{\mathrm{S}}$-groupoid structures on a $d p b_{\mathrm{T}^{-}}$chain $\langle A, \wedge, \vee, 0,1, \perp, \top\rangle$, i.e., we have two $r t_{\mathrm{S}}$-groupoids

$$
\boldsymbol{A}_{1}=\left\langle A, \wedge, \vee, \circ^{\boldsymbol{A}_{1}}, \backslash^{\boldsymbol{A}_{1}}, /^{\boldsymbol{A}_{1}}, 0,1, \perp, \top\right\rangle, \quad \boldsymbol{A}_{2}=\left\langle A, \wedge, \vee, \circ^{\boldsymbol{A}_{2}}, \backslash^{\boldsymbol{A}_{2}}, / \boldsymbol{A}^{\boldsymbol{A}_{2}}, 0,1, \perp, \top\right\rangle .
$$

Then we define on the same $d p b_{\mathrm{T}}$-chain an algebra $\boldsymbol{A}_{1} \wedge \boldsymbol{A}_{2}=\langle A, \wedge, \vee, \circ, \backslash, /, 0,1, \perp, \top\rangle$ as follows:

$$
a \circ b=\left(a \circ^{\boldsymbol{A}_{1}} b\right) \wedge\left(a \circ^{\boldsymbol{A}_{2}} b\right), \quad a \backslash b=\left(a \backslash^{\boldsymbol{A}_{1}} b\right) \vee\left(a \backslash^{\boldsymbol{A}_{2}} b\right), \quad a / b=\left(a /{ }^{\boldsymbol{A}_{1}} b\right) \vee\left(a /{ }^{\boldsymbol{A}_{2}} b\right) .
$$

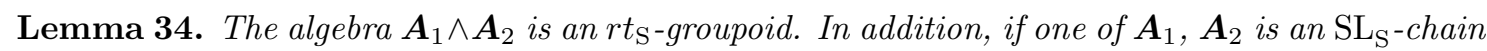
then $\boldsymbol{A}_{1} \wedge \boldsymbol{A}_{2}$ is an $\mathrm{SL}_{\mathrm{S}}$-chain as well.

Proof. First, $1 \circ a=\left(1 \circ^{\boldsymbol{A}_{1}} a\right) \wedge\left(1 \circ^{\boldsymbol{A}_{2}} a\right) \geq a \wedge a=a$. Similarly, $a \leq a \circ 1$. Further we have the following chain of equivalences:

$$
\begin{aligned}
a \circ b=\left(a \circ{ }^{\boldsymbol{A}_{1}} b\right) \wedge\left(a \circ{ }^{\boldsymbol{A}_{2}} b\right) \leq c & \text { iff } \quad a \circ^{\boldsymbol{A}_{1}} b \leq c \text { or } a \circ{ }^{\boldsymbol{A}_{2}} b \leq c \\
& \text { iff } b \leq a \backslash^{\boldsymbol{A}_{1}} c \text { or } b \leq a \backslash^{\boldsymbol{A}_{2}} c \\
& \text { iff } b \leq\left(a \backslash^{\boldsymbol{A}_{1}} c\right) \vee\left(a \backslash^{\boldsymbol{A}_{2}} c\right)=a \backslash c .
\end{aligned}
$$

Similarly we can prove $a \circ b \leq c$ iff $a \leq c / b$.

It is easy to see that commutativity is preserved by the construction of $\boldsymbol{A}_{1} \wedge \boldsymbol{A}_{2}$. To see that contraction is preserved, note that $a \circ a=\left(a \circ{ }^{\boldsymbol{A}_{1}} a\right) \wedge\left(a \circ \boldsymbol{A}_{2} a\right) \geq a \wedge a=a$. Thus $\boldsymbol{A}_{1} \wedge \boldsymbol{A}_{2}$ is an $r \ell_{\mathrm{S}}$-groupoid.

To see the additional part, assume without any loss of generality that $\boldsymbol{A}_{2}$ is an $\mathrm{SL}_{\mathrm{S}^{-}}$-chain. Then $a \circ^{\boldsymbol{A}_{2}} 1=a=1 \circ^{\boldsymbol{A}_{2}} a$. Thus we have $1 \circ a=\left(1 \circ^{\boldsymbol{A}_{1}} a\right) \wedge\left(1 \circ^{\boldsymbol{A}_{2}} a\right)=\left(1 \circ^{\boldsymbol{A}_{1}} a\right) \wedge a=a$ because $1 \circ^{\boldsymbol{A}_{1}} a \geq a$. Similarly, $a \circ 1=a$. 
Further we introduce a sort of extension construction. Let $\mathrm{S} \subseteq\{\mathrm{e}, \mathrm{c}, \mathrm{i}, \mathrm{o}\}$ and $\mathrm{T}=\mathrm{S} \backslash\{\mathrm{e}, \mathrm{c}\}$. Suppose we have a $d p b_{\mathrm{T}^{-}}$chain $\langle B, \wedge, \vee, 0,1, \perp, \top\rangle$, a subset $A \subseteq B$ such that $\{0,1, \perp, \top\} \subseteq A$, and an $r t_{\mathrm{S}}$-groupoid

$$
\boldsymbol{A}=\left\langle A, \wedge, \vee, \circ^{\boldsymbol{A}}, \backslash^{\boldsymbol{A}}, /^{\boldsymbol{A}}, 0,1, \perp, \top\right\rangle .
$$

Further, assume that there are a closure operator $\gamma$ and an interior operator $\sigma$ on $\langle B, \wedge, \vee\rangle$ such that $\gamma[B]=\sigma[B]=A$. This means that for every $b \in B$ we can find the least $a \in A$ such that $b \leq a$ (namely $\gamma(b)$ ) and the greatest $a^{\prime} \in A$ such that $a^{\prime} \leq b$ (namely $\left.\sigma(b)\right)$. We define an algebra $\boldsymbol{B}=\left\langle B, \wedge, \vee,{ }^{\boldsymbol{B}}, \backslash^{\boldsymbol{B}}, /^{\boldsymbol{B}}, 0,1, \perp, \top\right\rangle$ as follows:

$$
x \circ^{\boldsymbol{B}} y=\gamma(x) \circ^{\boldsymbol{A}} \gamma(y), \quad x /^{\boldsymbol{B}} y=\sigma(x) /^{\boldsymbol{A}} \gamma(y), \quad x \backslash^{\boldsymbol{B}} y=\gamma(x) \backslash^{\boldsymbol{A}} \sigma(y) .
$$

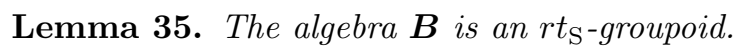

Proof. First, we prove that $\boldsymbol{B}$ is residuated. Suppose that $x \circ^{\boldsymbol{B}} y=\gamma(x) \circ^{\boldsymbol{A}} \gamma(y) \leq z$. Since $\gamma(x) \circ^{\boldsymbol{A}} \gamma(y)$ is $\sigma$-open, we have $\gamma(x) \circ^{\boldsymbol{A}} \gamma(y)=\sigma\left(\gamma(x) \circ^{\boldsymbol{A}} \gamma(y)\right) \leq \sigma(z)$. Consequently, $x \leq \gamma(x) \leq$ $\sigma(z) /{ }^{\boldsymbol{A}} \gamma(y)=z /{ }^{\boldsymbol{B}} y$. Conversely, suppose that $x \leq z /{ }^{\boldsymbol{B}} y=\bar{\sigma}(z) /{ }^{\boldsymbol{A}} \gamma(y)$. Since $\sigma(z) /{ }^{\boldsymbol{A}} \gamma(y)$ is $\gamma$-closed, we have $\gamma(x) \leq \gamma\left(\sigma(z) /{ }^{\boldsymbol{A}} \gamma(y)\right)=\sigma(z) /{ }^{\boldsymbol{A}} \gamma(y)$. Consequently, $x \circ^{\boldsymbol{B}} y=\gamma(x){ }^{\boldsymbol{A}} \gamma(y) \leq$ $\sigma(z) \leq z$. Analogously for the left division. Finally, note that

$$
1 \circ^{\boldsymbol{B}} x=\gamma(1) \circ^{\boldsymbol{A}} \gamma(x)=1 \circ^{\boldsymbol{A}} \gamma(x) \geq \gamma(x) \geq x .
$$

Similarly, $x \circ^{\boldsymbol{B}} 1 \geq x$. Thus $\boldsymbol{B}$ is an $r$-groupoid.

Next we have to show that $\boldsymbol{B}$ is in fact an $r t_{\mathrm{S}}$-groupoid. To see this, note that $\boldsymbol{B}$ is commutative if $\boldsymbol{A}$ is. If $\boldsymbol{A}$ is contractive then we have $x \circ^{\boldsymbol{B}} x=\gamma(x){ }^{\circ} \boldsymbol{A} \gamma(x) \geq \gamma(x) \geq x$ for any $x \in B$.

Finally, let $\mathrm{T} \subseteq\{\mathrm{i}, \mathrm{o}\}, \mathrm{S}=\mathrm{T} \cup\{\mathrm{e}, \mathrm{c}\}$, and $\boldsymbol{C}=\langle C, \wedge, \vee, 0,1, \mathrm{~T}, \perp\rangle$ a $d p b_{\mathrm{T}}$-chain. We will show that there is a greatest groupoid operation on $\boldsymbol{C}$ making $\boldsymbol{C}$ into an $\mathrm{SL}_{\mathrm{S}}$-chain $\boldsymbol{M}(\boldsymbol{C})=$ $\langle C, \wedge, \vee, \odot, \rightarrow, 0,1, \perp, \top\rangle$. We define

$$
x \odot y= \begin{cases}\top & \text { if } x, y>1 \\ \perp & \text { if } x=\perp \text { or } y=\perp \\ x \wedge y & \text { if } x, y \leq 1 \\ x \vee y & \text { otherwise }\end{cases}
$$

Lemma 36. The algebra $\boldsymbol{M}(\boldsymbol{C})=\langle C, \wedge, \vee, \odot, \rightarrow, 0,1, \perp, \top\rangle$ is an $\mathrm{SL}_{\mathrm{S}}$-chain, where $\rightarrow$ is the uniquely determined residual of $\odot$. Moreover, $\odot$ is the maximum among all groupoid operations ○ on $\boldsymbol{C}$ making it into an $\mathrm{SL}_{\mathrm{S}}$-chain w.r.t. the point-wise order, i.e., $x \circ y \leq x \odot y$ for all $x, y \in C$.

Proof. $\boldsymbol{M}(\boldsymbol{C})$ is clearly a $d p b_{\mathrm{T}^{-}}$chain. It is also easy to see that $\odot$ is commutative and contractive. Further, we have $1 \odot x=1 \wedge x=x$ if $x \leq 1$ and $1 \odot x=1 \vee x=x$ if $x>1$. Thus 1 is a neutral element for $\odot$. In order to show that $\odot$ is residuated, it suffices (due to commutativity of $\odot$ ) to show that for all $a \in C$ the map $f_{a}: C \rightarrow C$ defined by $f_{a}(x)=a \odot x$ is residuated. Depending on $a$, the map $f_{a}$ could be of a different shape. If $a \leq 1$ then

$$
f_{a}(x)= \begin{cases}a & \text { if } x \in[a, 1] \\ x & \text { otherwise. }\end{cases}
$$

If $a>1$ then

$$
f_{a}(x)= \begin{cases}\perp & \text { if } x=\perp \\ a & \text { if } \perp<x \leq 1 \\ \top & \text { if } x>1\end{cases}
$$

In both cases it is easy to see that $f_{a}$ is residuated (it is monotone and the inverse image of any principal downset is a principal downset). 
Let $\circ$ be a groupoid operation on $\boldsymbol{C}$ making it into an $\mathrm{SL}_{\mathrm{S}}$-chain. Since 1 is a neutral element for $\circ$, we must have $x \circ y \leq x \wedge y=x \odot y$ for $x, y \leq 1$. Further, for $\perp<x \leq 1$ and $y>1$ we must have $x \circ y \leq y=x \vee y=x \odot y$ and similarly $x \circ y \leq x \odot y$ for $x>1$ and $\perp<y \leq 1$. Since $\circ$ is residuated, it has to satisfy $\perp \circ x=x \circ \perp=\perp=\perp \odot x=x \odot \perp$. Finally, $x \circ y \leq \top=x \odot y$ for $x, y>1$. Thus $x \circ y \leq x \odot y$ for all $x, y \in C$.

Proof of Theorem 32. Let $\mathrm{S} \subseteq\{\mathrm{e}, \mathrm{c}, \mathrm{i}, \mathrm{o}\}, \mathrm{T}=\mathrm{S} \backslash\{\mathrm{e}, \mathrm{c}\}$. By Theorem 31 in order to show $\mathrm{S} \mathcal{Q C}$ for $\mathrm{SL}_{\mathrm{S}}^{\ell}$, it is sufficient to prove that each countable nontrivial $\mathrm{SL}_{\mathrm{S}}$-chain $\boldsymbol{A}$ can be embedded into a countably infinite dense $\mathrm{SL}_{\mathrm{S}}$-chain $\boldsymbol{D}$ because every countable infinite dense chain having a minimum $\perp$ and a maximum $T$ is order-isomorphic to $\mathrm{Q} \cap[0,1]$.

Suppose that we have an $\mathrm{SL}_{\mathrm{S}}$-chain $\boldsymbol{A}=\left\langle A, \wedge, \vee,{ }^{\boldsymbol{A}}, \backslash^{\boldsymbol{A}}, /^{\boldsymbol{A}}, 0,1, \perp, \top\right\rangle$ which is countable and nontrivial. Then its reduct $\langle A, \wedge, \vee, 0,1, \perp, \top\rangle$ forming a $d p b_{\mathrm{T}}$-chain can be extended to a countably infinite dense $d p b_{\mathrm{T}^{-}}$chain $\langle D, \wedge, \vee, 0,1, \perp, \top\rangle$ by Lemma 33 in such a way that there are a closure operator $\gamma$ and an interior operator $\sigma$ on $\langle D, \wedge, \vee, 0,1, \perp, \top\rangle$ such that $\gamma[D]=$ $\sigma[D]=A$. The next step is to extend the multiplication on the $\mathrm{SL}_{\mathrm{S}}$-chain $\boldsymbol{A}$ to $\boldsymbol{D}$. Applying Lemma 35 to our dense $d p b_{\mathrm{T}^{-}}$chain $\langle D, \wedge, \vee, 0,1, \perp, \top\rangle$, we obtain an $r t_{\mathrm{S}}$-groupoid $\boldsymbol{D}=$ $\left\langle D, \wedge, \vee, \circ^{\boldsymbol{D}}, \backslash^{\boldsymbol{D}}, /^{\boldsymbol{D}}, 0,1, \perp, \top\right\rangle$. However, ${ }^{\boldsymbol{D}}$ is not unital because 1 need not be a neutral element. In particular, $1 \circ^{D} x=\gamma(x) \geq x$, i.e., the result of $1 \circ^{\boldsymbol{D}} x$ could be greater than we need. Thus we have to further modify ${ }^{D} \boldsymbol{D}$. By Lemma 36 the $d p b_{\mathrm{T}^{-}}$chain $\langle D, \wedge, \vee, 0,1, \perp, \top\rangle$ also forms an $\mathrm{SL}_{\mathrm{T} \cup\{\mathrm{e}, \mathrm{c}\}}$-chain $\boldsymbol{M}(\boldsymbol{D})=\langle D, \wedge, \vee, \odot, \rightarrow, 0,1, \perp, \top\rangle$ such that $\odot$ is the maximum among all residuated groupoid operations on $\boldsymbol{D}$ having 1 as a neutral element. Thus it seems to be natural to lessen the values of ${ }^{\boldsymbol{D}} \boldsymbol{D}$ by a combination with $\odot$. Namely, $\boldsymbol{D} \wedge \boldsymbol{M}(\boldsymbol{D})=\langle D, \wedge, \vee, \circ, \backslash, /, 0,1, \perp, \top\rangle$ is an $\mathrm{SL}_{\mathrm{S}}$-chain by Lemma 34 .

Finally, we have to show that $\boldsymbol{A}$ can be embedded into $\boldsymbol{D} \wedge \boldsymbol{M}(\boldsymbol{D})$. We claim that the identity map from $A$ to $D$ is the desired embedding. Let $x, y \in A$. Then $\gamma(x)=\sigma(x)=x$ and $\gamma(y)=\sigma(y)=y$. By Lemma 36 we have $x \circ^{\boldsymbol{A}} y \leq x \odot y$. Thus

$$
x \circ y=\left(\gamma(x) \circ^{\boldsymbol{A}} \gamma(y)\right) \wedge(x \odot y)=\left(x \circ^{\boldsymbol{A}} y\right) \wedge(x \odot y)=x \circ^{\boldsymbol{A}} y .
$$

For the right division we have

$$
x / y=\left(\sigma(x) /{ }^{\boldsymbol{A}} \gamma(y)\right) \vee(y \rightarrow x)=\left(x /{ }^{\boldsymbol{A}} y\right) \vee(y \rightarrow x) .
$$

Using again Lemma 36 together with the commutativity of $\odot$, we obtain

$$
(y \rightarrow x) \circ^{\boldsymbol{A}} y \leq(y \rightarrow x) \odot y=y \odot(y \rightarrow x) \leq x .
$$

Thus by residuation $y \rightarrow x \leq x /{ }^{\boldsymbol{A}} y$. Consequently, we have $x / y=x /{ }^{\boldsymbol{A}} y$. Similarly, we can prove $x \backslash y=x \backslash^{\boldsymbol{A}} y$ which finishes the proof of the $\mathrm{S} \mathcal{Q C}$ for $\mathrm{SL}_{\mathrm{S}}^{\ell}$.

Now it is easy to extend this result to $\mathrm{S} \mathcal{R} C$ using the Dedekind-MacNeille completion and again Theorem 31. Let $\boldsymbol{A}$ be a $\mathrm{SL}_{\mathrm{S}}$-chain from $\mathcal{Q}$ and $\boldsymbol{A}^{\prime}$ its lattice reduct. Then, as was shown in [17], $\boldsymbol{A}$ can be embedded into an $\mathrm{SL}_{\mathrm{S}^{-}}$-algebra $\boldsymbol{B}$ whose lattice reduct is the DedekindMacNeille completion of $\boldsymbol{A}^{\prime}$. Since the Dedekind-MacNeille completion of the chain $Q \cap[0,1]$ is order-isomorphic to $[0,1]$, we are done.

Acknowledgements P. Cintula and R. Horčík were supported by grant P202/12/G061 of the Czech Science Foundation. P. Cintula was also supported by grant I143-G15 (LogICCC/LoMoReVI) of the ESF/Austrian Science Foundation (FWF). C. Noguera was supported by research contract 'Juan de la Cierva' JCI-2009-05453, the MICINN project TASSAT (TIN2010-20967-C04-01) and the FP7-PEOPLE-2009-IRSES project MaToMUVI (PIRSES-GA-2009-247584).

\section{References}

[1] A. R. Anderson and N. D. Belnap. Entailment: The Logic of Relevance and Necessity, volume 1. Princeton University Press, Princeton, 1975. 
[2] L. Běhounek and P. Cintula. Fuzzy logics as the logics of chains. Fuzzy Sets and Systems, 157(5):604-610, 2006.

[3] W. J. Blok and D. L. Pigozzi. Algebraizable Logics, volume 396 of Memoirs of the American Mathematical Society. American Mathematical Society, Providence, RI, 1989. Freely downloadable from http://orion.math.iastate.edu/dpigozzi/.

[4] M. Botur. A non-associative generalization of Hájek's BL-algebras. Fuzzy Sets and Systems, 178(1):24-37, 2011.

[5] S. Burris and H. Sankappanavar. A Course in Universal Algebra, volume 78 of Graduate Texts in Mathematics. Springer-Verlag, 1981.

[6] W. Buszkowski and M. Farulewski. Nonassociative Lambek calculus with additives and context free languages. In O. Grumberg, M. Kaminski, S. Katz, and S. Wintner, editors, Languages: From Formal to Natural. Essays Dedicated to Nissim Francez, volume 5533 of Lecture Notes in Computer Science. Springer, Berlin, 2009.

[7] R. Cignoli, F. Esteva, L. Godo, and A. Torrens. Basic fuzzy logic is the logic of continuous t-norms and their residua. Soft Computing, 4(2):106-112, 2000.

[8] P. Cintula, P. Hájek, and R. Horčík. Formal systems of fuzzy logic and their fragments. Annals of Pure and Applied Logic, 150(1-3):40-65, 2007.

[9] P. Cintula, P. Hájek, and C. Noguera, editors. Handbook of Mathematical Fuzzy Logic (in 2 volumes), volume 37, 38 of Studies in Logic, Mathematical Logic and Foundations. College Publications, London, 2011.

[10] P. Cintula and C. Noguera. The proof by cases property and its variants in structural consequence relations. To appear in Studia Logica.

[11] P. Cintula and C. Noguera. Implicational (semilinear) logics I: A new hierarchy. Archive for Mathematical Logic, 49(4):417-446, 2010.

[12] P. Cintula and C. Noguera. A general framework for mathematical fuzzy logic. In P. Cintula, P. Hájek, and C. Noguera, editors, Handbook of Mathematical Fuzzy Logic - Volume 1, volume 37 of Studies in Logic, Mathematical Logic and Foundations. 103-207, London, 2011.

[13] J. Czelakowski. Protoalgebraic Logics, volume 10 of Trends in Logic. Kluwer, Dordrecht, 2001.

[14] F. Esteva and L. Godo. Monoidal t-norm based logic: Towards a logic for left-continuous t-norms. Fuzzy Sets and Systems, 124(3):271-288, 2001.

[15] F. Esteva, L. Godo, P. Hájek, and F. Montagna. Hoops and fuzzy logic. Journal of Logic and Computation, 13(4):532-555, 2003.

[16] N. Galatos. Equational bases for joins of residuated-lattice varieties. Studia Logica, 76(2):227240, 2004.

[17] N. Galatos and P. Jipsen. Residuated frames with applications to decidability. To appear in the Transactions of the AMS.

[18] N. Galatos, P. Jipsen, T. Kowalski, and H. Ono. Residuated Lattices: An Algebraic Glimpse at Substructural Logics, volume 151 of Studies in Logic and the Foundations of Mathematics. Elsevier, Amsterdam, 2007.

[19] N. Galatos and H. Ono. Cut elimination and strong separation for substructural logics: An algebraic approach. Annals of Pure and Applied Logic, 161(9):1097-1133, 2010. 
[20] J.-Y. Girard. Linear logic. Theoretical Computer Science, 50(1):1-102, 1987.

[21] P. Hájek. Metamathematics of Fuzzy Logic, volume 4 of Trends in Logic. Kluwer, Dordrecht, 1998.

[22] U. Höhle. Commutative, residuated l-monoids. In U. Höhle and E. P. Klement, editors, NonClassical Logics and Their Applications to Fuzzy Subsets, pages 53-106. Kluwer, Dordrecht, 1995.

[23] R. Horčík. Algebraic semantics: Semilinear FL-algebras. In P. Cintula, P. Hájek, and C. Noguera, editors, Handbook of Mathematical Fuzzy Logic - Volume 1, volume 37 of Studies in Logic, Mathematical Logic and Foundations. 283-353, London, 2011.

[24] S. Jenei and F. Montagna. A proof of standard completeness for Esteva and Godo's logic MTL. Studia Logica, 70(2):183-192, 2002.

[25] S. Jenei and F. Montagna. A proof of standard completeness for non-commutative monoidal t-norm logic. Neural Network World, 13(5):481-489, 2003.

[26] J. Lambek. On the calculus of syntactic types. American Mathematical Monthly, 65(3):154170,1958

[27] J. Lambek. The mathematics of sentence structure. In R. Jakobson, editor, Structure of Language and Its Mathematical Aspects, pages 166-178. AMS, Providence, 1961.

[28] G. Metcalfe and F. Montagna. Substructural fuzzy logics. Journal of Symbolic Logic, $72(3): 834-864,2007$.

[29] F. Paoli. Substructural Logics: A Primer, volume 13 of Trends in Logic. Kluwer, Dordrecht, 2002 .

[30] J. G. Raftery. A characterization of varieties. Algebra Universalis, 45(4):449-450, 2001.

[31] G. Restall. An Introduction to Substructural Logics. Routledge, New York, 2000.

[32] P. Schroeder-Heister and K. Dosen, editors. Substructural Logics, volume 2 of Studies in Logic and Computation. Oxford University Press, Oxford, 1994.

[33] S. Wang and B. Zhao. HpsUL is not the logic of pseudo-uninorms and their residua. Logic Journal of the Interest Group of Pure and Applied Logic, 17(4):413-419, 2009.

\section{A The proof of Theorem 9}

To prove one direction we only need to know the derivability of the new rules of $\mathcal{A S}$ in SL (all its axioms are either shown to be theorems of SL in the preliminaries or can be proved easily e.g. in the Gentzen calculus for SL). Conversely, we show that $\mathcal{A S}$ proves all axioms and rules of SL.

- $\mathrm{SL}$ proves $(\alpha)$ :
(a) $\vdash \chi \rightarrow(\psi \rightarrow \psi \& \chi)$
(b) $\chi \vdash \psi \rightarrow \psi \& \chi$
(a) and (MP)
(c) $\chi \vdash \varphi \& \psi \rightarrow \varphi \&(\psi \& \chi)$
$\left(\mathrm{P}_{\mathrm{SL}} 8\right),(\mathrm{b})$, and (MP)

- SL proves $\left(\alpha^{\prime}\right)$ :
(a) $\vdash \chi \rightarrow(\varphi \rightarrow \varphi \& \chi)$
(b) $\chi \vdash \varphi \rightarrow \varphi \& \chi$
(a) and (MP)
(c) $\chi \vdash \varphi \& \psi \rightarrow(\varphi \& \chi) \& \psi$
$\left(\mathrm{P}_{\mathrm{SL}} 9\right),(\mathrm{b})$, and (MP) 
- $\mathrm{SL}$ proves $(\beta)$ :
(a) $\vdash \chi \rightarrow(\varphi \& \psi \rightarrow(\varphi \& \psi) \& \chi)$
$\left(\operatorname{Adj}_{\&}\right)$
(b) $\quad \chi \vdash \varphi \& \psi \rightarrow(\varphi \& \psi) \& \chi$
(a) and (MP)
(c) $\chi \vdash \psi \rightarrow(\varphi \rightarrow(\varphi \& \psi) \& \chi)$
(b) and (Res)

- $\mathrm{SL}$ proves $\left(\beta^{\prime}\right)$ :

(a) $\chi \vdash \varphi \rightarrow(\psi \rightarrow(\psi \& \varphi) \& \chi)$

(b) $\quad \vee^{\vdash} \psi \rightarrow(\varphi \rightsquigarrow(\psi \& \varphi) \& \chi)$

(a) and $\left(\mathrm{E}_{\rightsquigarrow 1}\right)$

- $\mathcal{A S}$ proves $\chi \rightarrow \varphi, \varphi \rightarrow \psi \vdash \chi \rightarrow \psi(\mathrm{T})$ :

(a) $\vdash(\chi \rightarrow(\chi \&(\chi \rightarrow \varphi)) \&(\varphi \rightarrow \psi)) \rightarrow(\chi \rightarrow \psi)$

(b) $\varphi \rightarrow \psi \vdash(\chi \rightarrow \varphi) \rightarrow(\chi \rightarrow(\chi \&(\chi \rightarrow \varphi)) \&(\varphi \rightarrow \psi))$

(c) $\chi \rightarrow \varphi, \varphi \rightarrow \psi \vdash(\chi \rightarrow(\chi \&(\chi \rightarrow \varphi)) \&(\varphi \rightarrow \psi))$

(b) and (MP)

(d) $\chi \rightarrow \varphi, \varphi \rightarrow \psi \vdash \chi \rightarrow \psi$ (a), (c), and (MP)

- $\mathcal{A S}$ proves $\varphi \rightarrow \psi \vdash(\chi \rightarrow \varphi) \rightarrow(\chi \rightarrow \psi)(\mathrm{Pf})$ :

(a) $\vdash(\chi \rightarrow(\chi \&(\chi \rightarrow \varphi)) \&(\varphi \rightarrow \psi)) \rightarrow(\chi \rightarrow \psi)$

(b) $\varphi \rightarrow \psi \vdash(\chi \rightarrow \varphi) \rightarrow(\chi \rightarrow(\chi \&(\chi \rightarrow \varphi)) \&(\varphi \rightarrow \psi))$

(c) $\varphi \rightarrow \psi \vdash(\chi \rightarrow \varphi) \rightarrow(\chi \rightarrow \psi)$

(a), (b), and (T)

- $\mathcal{A S}$ proves $\varphi \rightarrow \psi \vdash(\chi \rightsquigarrow \varphi) \rightarrow(\chi \rightsquigarrow \psi)\left(\mathrm{Pf}_{\rightsquigarrow}\right)$ :
(a) $\vdash(\chi \rightsquigarrow((\chi \rightsquigarrow \varphi) \& \chi) \&(\varphi \rightarrow \psi)) \rightarrow(\chi \rightsquigarrow \psi)$
(b) $\varphi \rightarrow \psi \vdash(\chi \rightsquigarrow \varphi) \rightarrow(\chi \rightsquigarrow((\chi \rightsquigarrow \varphi) \& \chi) \&(\varphi \rightarrow \psi))$
(c) $\varphi \rightarrow \psi \vdash(\chi \rightsquigarrow \varphi) \rightarrow(\chi \rightsquigarrow \psi)$

(a), (b), and (T)

- $\mathcal{A S}$ proves $\varphi \rightarrow(\psi \rightarrow \chi) \vdash \psi \& \varphi \rightarrow \chi\left(\operatorname{Res}_{1}\right)$ :

(a) $\vdash \psi \&(\varphi \&(\varphi \rightarrow(\psi \rightarrow \chi))) \rightarrow \chi$

(b) $\varphi \rightarrow(\psi \rightarrow \chi) \vdash \psi \& \varphi \rightarrow \psi \&(\varphi \&(\varphi \rightarrow(\psi \rightarrow \chi)))$

(c) $\varphi \rightarrow(\psi \rightarrow \chi) \vdash \psi \& \varphi \rightarrow \chi$

(a), (b), and (T)

- $\mathcal{A S}$ proves $\varphi \rightarrow(\psi \rightsquigarrow \chi) \vdash \varphi \& \psi \rightarrow \chi\left(\operatorname{Res}_{\rightsquigarrow 1}\right)$ :
(a) $\vdash(\varphi \&(\varphi \rightarrow(\psi \rightsquigarrow \chi))) \& \psi \rightarrow \chi$
(b) $\varphi \rightarrow(\psi \rightsquigarrow \chi) \vdash \varphi \& \psi \rightarrow(\varphi \&(\varphi \rightarrow(\psi \rightsquigarrow \chi))) \& \psi$
(c) $\varphi \rightarrow(\psi \rightsquigarrow \chi) \vdash \varphi \& \psi \rightarrow \chi$

$\left(\operatorname{Res}_{\rightsquigarrow}^{\prime}\right)$

(a), (b), and (T)

- $\mathcal{A S}$ proves $\psi \& \varphi \rightarrow \chi \vdash \varphi \rightarrow(\psi \rightarrow \chi)\left(\operatorname{Res}_{2}\right)$ :
(a) $\psi \& \varphi \rightarrow \chi \vdash(\psi \rightarrow \psi \& \varphi) \rightarrow(\psi \rightarrow \chi)$
(b) $\psi \& \varphi \rightarrow \chi \vdash(\varphi \rightarrow(\psi \rightarrow \psi \& \varphi)) \rightarrow(\varphi \rightarrow(\psi \rightarrow \chi))$
(c) $\vdash \varphi \rightarrow(\psi \rightarrow \psi \& \varphi)$
(d) $\psi \& \varphi \rightarrow \chi \vdash \varphi \rightarrow(\psi \rightarrow \chi)$

(a), (Pf), and (MP)

(b), (c), and (MP)

- $\mathcal{A S}$ proves $\psi \& \varphi \rightarrow \chi \vdash \psi \rightarrow(\varphi \rightsquigarrow \chi)\left(\operatorname{Res}_{\rightsquigarrow 2}\right)$ :
(a) $\quad \psi \& \varphi \rightarrow \chi \vdash(\varphi \rightsquigarrow \psi \& \varphi) \rightarrow(\varphi \rightsquigarrow \chi)$
(b) $\quad \psi \& \varphi \rightarrow \chi \vdash(\psi \rightarrow(\varphi \rightsquigarrow \psi \& \varphi)) \rightarrow(\psi \rightarrow(\varphi \rightsquigarrow \chi))$
(c) $\vdash \psi \rightarrow(\varphi \rightsquigarrow \psi \& \varphi)$
(d) $\psi \& \varphi \rightarrow \chi \vdash \psi \rightarrow(\varphi \rightsquigarrow \chi)$

(a), (Pf), and (MP)

$\left(\operatorname{Adj}_{\& \rightsquigarrow)}\right)$

(b), (c), and (MP)

- $\mathcal{A S}$ proves $\psi \rightarrow(\varphi \rightarrow \chi) \vdash \varphi \rightarrow(\psi \rightsquigarrow \chi)\left(\mathrm{E}_{\rightsquigarrow 1}\right)$ :
(a) $\psi \rightarrow(\varphi \rightarrow \chi) \vdash \varphi \& \psi \rightarrow \chi$
(b) $\psi \rightarrow(\varphi \rightarrow \chi) \vdash \varphi \rightarrow(\psi \rightsquigarrow \chi)$

$\left(\operatorname{Res}_{1}\right)$

(a) and $\left(\operatorname{Res}_{\rightsquigarrow 2}\right)$ 
- $\mathcal{A S}$ proves $\varphi \rightarrow(\psi \rightsquigarrow \chi) \vdash \psi \rightarrow(\varphi \rightarrow \chi)\left(\mathrm{E}_{\rightsquigarrow 2}\right)$ :
(a) $\varphi \rightarrow(\psi \rightsquigarrow \chi) \vdash \varphi \& \psi \rightarrow \chi$
$\left(\operatorname{Res}_{\rightsquigarrow 1}\right)$
(b) $\varphi \rightarrow(\psi \rightsquigarrow \chi) \vdash \psi \rightarrow(\varphi \rightarrow \chi)$
(b) and $\left(\operatorname{Res}_{2}\right)$

- $\mathcal{A S}$ proves $\varphi \rightarrow \varphi$ (R): (Push), (Pop), and (T).

- $\mathcal{A S}$ proves $\overline{1} \rightarrow(\varphi \rightarrow \varphi)\left(\mathrm{R}^{\prime}\right)$ :

(a) $\varphi \rightarrow \varphi \vdash \overline{1} \rightarrow(\varphi \rightarrow \varphi)$

(Push) and (MP)

(b) $\vdash \overline{1} \rightarrow(\varphi \rightarrow \varphi)$

(R) and (a)

- $\mathcal{A S}$ proves $\overline{1}(\overline{1})$ :
(a) $\vdash(\overline{1} \rightarrow \overline{1}) \rightarrow \overline{1}$
(b) $\vdash \overline{1}$

(R), (a), and (MP)

- $\mathcal{A S}$ proves $\varphi \rightarrow((\varphi \rightsquigarrow \psi) \rightarrow \psi)\left(\right.$ As $\left._{\ell \ell}\right)$ :
(a) $\vdash(\varphi \rightsquigarrow \psi) \rightarrow(\varphi \rightsquigarrow \psi)$
(b) $\vdash \varphi \rightarrow((\varphi \rightsquigarrow \psi) \rightarrow \psi)$

(a) and $\left(\mathrm{E}_{\rightsquigarrow 2}\right)$

- $\mathcal{A S}$ proves $\varphi \rightarrow \psi \vdash(\psi \rightarrow \chi) \rightarrow(\varphi \rightarrow \chi)$ (Sf):
(a) $\vdash(\psi \rightarrow \chi) \rightarrow(\psi \rightarrow \chi)$
(b) $\vdash \psi \rightarrow((\psi \rightarrow \chi) \rightsquigarrow \chi)$
(c) $\varphi \rightarrow \psi \vdash \varphi \rightarrow((\psi \rightarrow \chi) \rightsquigarrow \chi)$
(d) $\varphi \rightarrow \psi \vdash(\psi \rightarrow \chi) \rightarrow(\varphi \rightarrow \chi)$

(a) and $\left(E_{\rightsquigarrow 1}\right)$ (Pf), (b), and (T)

(c) and $\left(\mathrm{E}_{\rightsquigarrow 2}\right)$

- $\mathcal{A S}$ proves $\varphi \vdash(\varphi \rightarrow \psi) \rightarrow \psi(\mathrm{As})$ :
(a) $\varphi \vdash \overline{1} \rightarrow \varphi$
(b) $\varphi \vdash(\varphi \rightarrow \psi) \rightarrow(\overline{1} \rightarrow \psi)$
(c) $\vdash(\overline{1} \rightarrow \psi) \rightarrow \psi$
(d) $\varphi \vdash(\varphi \rightarrow \psi) \rightarrow \psi$

(Push) and (MP)

(a) and (Sf)

(Pop)

(b), (c), and (T)

- $\mathcal{A S}$ proves $\varphi, \psi \vdash \varphi \wedge \psi(\mathrm{Adj})$ :
(a) $\varphi \vdash \varphi \wedge \overline{1}$
(b) $\psi \vdash \psi \wedge \overline{1}$
(c) $\vdash \psi \wedge \overline{1} \rightarrow(\varphi \wedge \overline{1} \rightarrow(\varphi \wedge \overline{1}) \&(\psi \wedge \overline{1}))$
(d) $\varphi, \psi \vdash(\varphi \wedge \overline{1}) \&(\psi \wedge \overline{1})$
(e) $\vdash(\varphi \wedge \overline{1}) \&(\psi \wedge \overline{1}) \rightarrow \varphi \wedge \psi$
(f) $\varphi, \psi \vdash \varphi \wedge \psi$

- $\mathcal{A S}$ proves $\varphi \rightsquigarrow \psi \vdash \varphi \rightarrow \psi\left(\mathrm{Symm}_{1}\right)$ :
(a) $\varphi \rightsquigarrow \psi \vdash \overline{1} \rightarrow(\varphi \rightsquigarrow \psi)$
(b) $\varphi \rightsquigarrow \psi \vdash \varphi \rightarrow(\overline{1} \rightarrow \psi)$
(c) $\vdash(\overline{1} \rightarrow \psi) \rightarrow \psi$
(d) $\varphi \rightsquigarrow \psi \vdash \varphi \rightarrow \psi$

(Push) and (MP) (a) and $\left(\mathrm{E}_{\rightsquigarrow 2}\right)$

(Pop)

(b), (c), and (T)

- $\mathcal{A S}$ proves $(\varphi \rightsquigarrow \chi) \wedge(\psi \rightsquigarrow \chi) \rightarrow(\varphi \vee \psi \rightsquigarrow \chi)\left(\vee 3_{\rightsquigarrow}\right)$ :
(a) $\vdash(\varphi \rightsquigarrow \chi) \wedge(\psi \rightsquigarrow \chi) \rightarrow(\varphi \rightsquigarrow \chi)$
(b) $\vdash \varphi \rightarrow((\varphi \rightsquigarrow \chi) \wedge(\psi \rightsquigarrow \chi) \rightarrow \chi)$
(c) $\vdash \psi \rightarrow((\varphi \rightsquigarrow \chi) \wedge(\psi \rightsquigarrow \chi) \rightarrow \chi)$
(d) $\vdash \varphi \vee \psi \rightarrow((\varphi \rightsquigarrow \chi) \wedge(\psi \rightsquigarrow \chi) \rightarrow \chi)$
(e) $\vdash(\varphi \rightsquigarrow \chi) \wedge(\psi \rightsquigarrow \chi) \rightarrow(\varphi \vee \psi \rightsquigarrow \chi)$

analogously

(Adj), (V3), (MP)

$\left(\mathrm{E}_{\rightsquigarrow 1}\right)$ 